\title{
Nutlin-3 enhances the bortezomib sensitivity of p53-defective cancer cells by inducing paraptosis
}

\author{
Dong Min Lee ${ }^{1,2}$, In Young Kim ${ }^{1,2}$, Min Ji Seo ${ }^{1,2}$, Mi Ri Kwon ${ }^{1,2}$ and Kyeong Sook Choi ${ }^{1,2}$ \\ The proteasome inhibitor, bortezomib, is ineffective against many solid tumors. Nutlin-3 is a potent antagonist of human \\ homolog of murine double minute $2 /$ p53 interaction exhibiting promising therapeutic anti-cancer activity. In this study, we show \\ that treatment of various p53-defective bortezomib-resistant solid tumor cells with bortezomib plus nutlin-3 induces paraptosis, \\ which is a cell death mode accompanied by dilation of the endoplasmic reticulum (ER) and mitochondria. Bortezomib alone \\ did not markedly alter cellular morphology, and nutlin-3 alone induced only a transient mitochondrial dilation. However, \\ bortezomib/nutlin-3 co-treatment triggered the progressive fusion of swollen ER and the formation of megamitochondria, \\ leading to cell death. Mechanistically, proteasomal-impairment-induced ER stress, CHOP upregulation and disruption of $\mathrm{Ca}^{2+}$ \\ homeostasis were found to be critically involved in the bortezomib/nutlin-3-induced dilation of the ER. Our results further \\ suggest that mitochondrial unfolded protein stress may play an important role in the mitochondrial dilation observed during \\ bortezomib/nutlin-3-induced cell death. Collectively, these findings suggest that bortezomib/nutlin-3 perturbs proteostasis, \\ triggering ER/mitochondria stress and irrecoverable impairments in their structure and function, ultimately leading to paraptotic \\ cell death.
}

Experimental \& Molecular Medicine (2017) 49, e365; doi:10.1038/emm.2017.112; published online 11 August 2017

\section{INTRODUCTION}

Proteasome-specific inhibitors have positive clinical benefits for cancer therapy. Bortezomib (PS341, Velcade), the first FDA-approved proteasome inhibitor (PI), is currently used to treat newly diagnosed and relapsed multiple myeloma and mantle cell lymphoma (MCL). ${ }^{1,2}$ Although bortezomib improves clinical outcomes when used as a single agent, most patients who do not respond to this drug almost uniformly relapse. ${ }^{3,4}$ Moreover, the clinical response to bortezomib has proven unsatisfactory in other hematologic malignancies and in solid tumors. ${ }^{3,5}$ Therefore, we need to develop clinically applicable approaches that will allow us to overcome the resistance of cancer cells to PIs and extend the activity of such agents to address a broader spectrum of tumors.

Nutlin-3 is a small-molecule antagonist of human homolog of murine double minute 2 (HDM2). It binds in the p53-binding pocket of HDM2 to block the HDM2-directed degradation of p53. ${ }^{6,7}$ The ability of nutlin-3 to restore the apoptotic response requires the presence of a p53 that is capable of transactivating its target genes; thus, nutlin-3 is believed to work best on tumors with wild-type p53. ${ }^{6,8}$
However, studies have also identified p53-independent effects of nutlin-3, ${ }^{9-13}$ further broadening its potential therapeutic range. For example, nutlin-3 was found to suppress cell growth and induce apoptosis in the absence of wild-type p53 via the p53 homolog, p73. ${ }^{9,10}$ In addition, nutlin-3 has been shown to sensitize p53-defective cancer cells to various anti-cancer agents, including radiation, ${ }^{11}$ doxorubicin, ${ }^{12}$ and arsenic trioxide. ${ }^{13}$

As defects in apoptotic signaling pathways (including those involving p53) are known to contribute to cancer development and therapeutic resistance in many types of malignant tumors, ${ }^{14,15}$ strategies to induce non-apoptotic cell death in such tumors may have considerable merit. Paraptosis (para $=$ next to or related to, and apoptosis) is a cell death mode that is characterized by: dilation of the ER and/or mitochondria; the requirement for new gene transcription and translation; and the lack of characteristic apoptotic features, such as apoptotic body formation, chromatin condensation, DNA fragmentation and caspase dependency. ${ }^{16,17}$ Recent work has shown that paraptosis is associated with the perturbation of cellular proteostasis (due to proteasome

\footnotetext{
${ }^{1}$ Department of Biochemistry, Ajou University School of Medicine, Suwon, Korea and ${ }^{2}$ BK21 Plus Program, Department of Biomedical Sciences, Ajou University School of Medicine, Suwon, Korea

Correspondence: Professor KS Choi, Department of Biochemistry, Department of Biomedical Sciences, Ajou University School of Medicine, Suwon 443-380, Korea.

E-mail: kschoi@ajou.ac.kr

Received 16 August 2016; revised 13 January 2017; accepted 5 March 2017
} 
inhibition and disruption of sulfhydryl homeostasis), ${ }^{18-24}$ generation of reactive oxygen species, ${ }^{18,24-26}$ and/or imbalanced homeostasis of ions (for example, $\mathrm{Ca}^{2+}$ and $\mathrm{K}^{+}$). ${ }^{19,20,24,27,28}$ However, the mechanisms underlying paraptosis, particularly the signals responsible for dilation of the ER and mitochondria, are still not clearly understood.

In the present study, we show that subtoxic doses of nutlin-3 can effectively overcome the bortezomib resistance of various p53-defective cancer cells by inducing the progressive swelling and fusion of the ER, the formation of megamitochondria, and eventual cell death (that is, paraptosis). Mechanistically, we found that proteasomal-impairment-induced ER stress, CHOP upregulation and disruption of $\mathrm{Ca}^{2+}$ homeostasis were critically involved in the ER dilation induced by bortezomib/nutlin-3. In addition, our data show that the mitochondrial unfolded protein stress response may contribute to the mitochondrial dilation induced by this combined treatment. We speculate that bortezomib/nultin-3 irreversibly perturbs proteostasis, leading to fatal impairments in the structures/functions of both the ER and mitochondria. Therefore, the use of bortezomib/nutlin-3 to induce paraptosis may offer a two-pronged attack strategy for killing resistant cancer cells and improving the effectiveness of this proteasome inhibitor as a therapeutic anti-cancer agent.

\section{MATERIALS AND METHODS}

\section{Chemicals and antibodies}

The chemicals used in this study were cycloheximide (CHX), MG132, 1,2-bis (o-aminophenoxy) ethane- $\mathrm{N}, \mathrm{N}, \mathrm{N}^{\prime}, \mathrm{N}^{\prime}$-tetraacetic acid (BAPTA) and 1,2-bis (o-aminophenoxy) ethane- $\mathrm{N}, \mathrm{N}, \mathrm{N}^{\prime}, \mathrm{N}^{\prime}$-tetraacetic acid acetoxymethyl ester (BAPTA-AM) (Sigma-Aldrich, St Louis, MO, USA); Nutlin-3 (TOCRIS, Avonmouth, Bristol, UK); Rhod-2-acetoxymethyl ester (Rhod-2-AM), Fluo-3-acetoxymethyl ester (Fluo-3-AM), MitoTracker-Red (MTR), MitoTracker-Green (MTG), calceinacetoxymethyl ester (calcein-AM), ethidium homodimer-1 (EthD-1), and 4',6-diamidino-2-phenylindole (DAPI) (Molecular Probe, Eugene, OR, USA); Ru360 (Calbiochem, Darmstadt, Germany); bortezomib, and carfilzomib (Selleckchem, Houston, TX, USA). The antibodies used in this study were anti- $\beta$-actin, ubiquitin, ATF4, mitochondrial $\mathrm{Ca}^{2+}$ uniporter (MCU), HDM2 and p53 (Santa Cruz Biotechnology, Santa Cruz, CA, USA); CHOP/GADD153, GRP78, and mtHsp70 (Cell Signaling Technology, Danvers, MA, USA); cytochrome oxidase subunit II (COX II) (Invitrogen, Grand Island, NY, USA); protein disulfide isomerase (PDI) (Enzo Life Sciences, Farmingdale, NY, USA); rabbit IgG HRP, mouse IgG HRP and goat IgG HRP (Molecular Probe).

\section{Cell culture}

The MDA-MB 435S (breast cancer), DLD-1 (colon cancer), HeLa (cervical cancer), T98G (glioblastoma), HCT116 (colon cancer), MCF-10A (normal breast) and CCD-841CoN (colon epithelial) cells were purchased from American Type Culture Collection (ATCC, Manassas, VA, USA). Cells were cultured in DMEM supplemented with $10 \%$ fetal bovine serum (FBS) and antibiotics (GIBCO-BRL, Grand Island, NY, USA) and incubated in $5 \% \mathrm{CO}_{2}$ at $37^{\circ} \mathrm{C}$.

\section{Determination of cellular viability using calcein-AM and} EthD-1 (live/dead assay)

Cells $\left(5 \times 10^{4}\right.$ cells $)$ were cultured in 24 -well plates and treated as indicated. For measurement of cellular viability, $2 \mu \mathrm{M}$ calcein-AM, a green fluorescent indicator of the intracellular esterase activity of cells, and $4 \mu \mathrm{M}$ EthD-1, a red fluorescent indicator of membrane damaged (dead) cells, were added to each well, and the plates were incubated for $5 \mathrm{~min}$ in $5 \%$ $\mathrm{CO}_{2}$ at $37^{\circ} \mathrm{C}$. Cells were then observed under a fluorescence microscope (Axiovert 200M; Carl Zeiss, Oberkochen, Germany) equipped with Zeiss filter sets \#46 and \#64HE. Viable cells, corresponding to those that exclusively exhibited green fluorescence, were counted in five fields per well at $\times 200$ magnification. Only exclusively green cells were counted as live because bicolored (green and red) cells cannot be unambiguously assigned to live or dead groups. The percentage of live cells (live \%), calculated as green cells/(green+red+bicolored cells), was normalized to that of untreated control cells $(100 \%)$.

\section{Examination of the stable cell lines expressing the fluorescence specifically in mitochondria or the endoplasmic reticulum}

To establish the stable cell lines expressing the fluorescence specifically in mitochondria or the ER, MDA-MB 435 S cells were transfected with the pEYFP-Mito or pEYFP-ER vector (Clontech, Mountain View, CA, USA). Stable cell lines expressing pEYFP-Mito or pEYFP-ER (YFP-Mito or YFP-ER) were selected with fresh medium containing $500 \mu \mathrm{g} \mathrm{ml}^{-1}$ G418 (Calbiochem). To quantitatively measure the dilation of the ER and mitochondria induced by treatment with bortezomib and/or nutlin-3, we analyzed the average width of the vacuoles originated from mitochondria and the ER in YFP-Mito cells and YFP-ER cells using AxioVision Rel. 4.8 software (Zeiss). More than 200 clearly identifiable vacuoles derived from the ER in 50 YFP-ER cells and more than 200 clearly identifiable vacuoles derived from mitochondria in 50 YFP-Mito cells per experiment were randomly selected and measured in three independent experiments.

\section{Western blotting}

Cells were washed in PBS and lysed in boiling sodium dodecyl sulfate/polyacrylamide gel electrophoresis (SDS-PAGE) sample buffer (62.5 mM Tris ( $\mathrm{pH}$ 6.8), 1\% SDS, 10\% glycerol and 5\% $\beta$-mercaptoethanol). The lysates were boiled for $5 \mathrm{~min}$, separated by SDS-PAGE, and transferred to an Immobilon membrane (Millipore, Bredford, MA, USA). After blocking nonspecific binding sites for $1 \mathrm{~h}$ using 5\% skim milk, membranes were incubated for $2 \mathrm{~h}$ with specific Abs. Membranes were then washed three times with TBST and incubated further for $1 \mathrm{~h}$ with horseradish peroxidase-conjugated anti-rabbit, anti-mouse or anti-goat antibody. Visualization of protein bands was accomplished using ECL (Advansta, Menlo Park, CA, USA). The representative results from at least three independent experiments are shown.

\section{Immunocytochemistry}

After treatments, cells were fixed with acetone/methanol (1:1) for $5 \mathrm{~min}$ at $-20^{\circ} \mathrm{C}$, blocked in $5 \%$ BSA in PBS for $30 \mathrm{~min}$, and incubated overnight at $4{ }^{\circ} \mathrm{C}$ with primary antibody (anti-COX II (1:500, mouse, Invitrogen), anti-PDI (1:500, rabbit, Enzo Life Sciences, Farmingdale, NY, USA), anti-mtHsp70 (1:250, rabbit, Cell Signaling Technology)) diluted in PBS. And then slides were washed three times in PBS, incubated for $1 \mathrm{~h}$ at room temperature with anti-rabbit or anti-mouse Alexa Fluor 488 or 594 (1:500, Molecular Probe), and mounted with ProLong Gold antifade mounting reagent (Molecular Probe). 
Cell staining was visualized with a fluorescence microscope using Zeiss filter sets \#46 and \#64HE (excitation band pass, 598/25 nm; emission band pass, $647 / 70 \mathrm{~nm}$ ). Colocalization of mtHsp70 and COX II following staining with anti-rabbit or anti-mouse Alexa Fluor 488 or 594 , respectively, was analyzed by qualitative line scans profiles using AxioVision Rel. 4.8 software (Zeiss).

\section{Measurement of cytosolic and mitochondrial $\mathrm{Ca}^{2+}$ levels}

To measure cytosolic $\left[\mathrm{Ca}^{2+}\right]_{\mathrm{c}}$ levels, treated cells were incubated with $1 \mu \mathrm{M}$ Fluo-3-AM at $37^{\circ} \mathrm{C}$ for $20 \mathrm{~min}$, washed with HBSS (without $\mathrm{Ca}^{2+}$ or $\mathrm{Mg}^{2+}$ ), and analyzed immediately by flow cytometry, or visualized by the fluorescence microscopy using Zeiss filter sets \#46. To measure mitochondrial $\left[\mathrm{Ca}^{2+}\right]_{\mathrm{mt}}$ levels, treated cells were incubated with $1 \mu \mathrm{M}$ Rhod-2-AM at $4{ }^{\circ} \mathrm{C}$ for $30 \mathrm{~min}$, washed with HBSS (without $\mathrm{Ca}^{2+}$ or $\mathrm{Mg}^{2+}$ ), further incubated with $\mathrm{HBSS}$ at $37^{\circ} \mathrm{C}$ for $20 \mathrm{~min}$, and then analyzed by flow cytometry. To confirm the mitochondrial localization of the Rhod-2 probe, cells were loaded with $1 \mu \mathrm{M}$ Rhod-2AM in HBSS (without $\mathrm{Ca}^{2+}$ or $\mathrm{Mg}^{2+}$ ) for 30 min at $4{ }^{\circ} \mathrm{C}$, then washed with HBSS, loaded with $100 \mathrm{~nm}$ MTG for $10 \mathrm{~min}$ in HBSS and visualized by the fluorescence microscopy using Zeiss filter sets \#46 and $\# 20$.

\section{Small interfering RNA- or small hairpin RNA-mediated knockdown of proteins}

The small interfering RNA (siRNA) duplexes of MCU (catalog no. L-015519-02) were purchased from Dharmacon (Lafeyette, CO, USA). Negative Universal Control (Invitrogen) was used as the control. After annealing of the pairs of siRNA oligos, cells were transfected with siRNA oligonucleotides. For the knockdown experiments using CHOP-targeting shRNA, HEK293TN cells were transfected with the plasmid containing the non-targeting shRNA $(\mathrm{SHC} 002 \mathrm{~V}$, Sigma-Aldrich) or the plasmid containing CHOP-targeting shRNA (TRCN0000364328, Sigma-Aldrich), together with pMD2.G (the envelope plasmid) and pPsAX2.0 plasmid (the packaging plasmid) using TransIT-2020 transfection reagents (Mirus Bio LLC, Madison, WI, USA) according to the manufacturer's instructions. After $48 \mathrm{~h}$ of lentiviral particle production, MDA-MB 435S cells were infected with the filtered lentiviral medium (derived from HEK293TN cultures) supplemented with $10 \mu \mathrm{g} \mathrm{ml}^{-1}$ polybrene. To confirm successful siRNA- or shRNA-mediated knockdown, Western blotting of the proteins of interest was performed.

\section{Transmission electron microscopy}

Cells were prefixed in Karnovsky's solution (1\% paraformaldehyde, $2 \%$ glutaraldehyde, $2 \mathrm{~mm}$ calcium chloride, $0.1 \mathrm{~m}$ cacodylate buffer, $\mathrm{pH}$ 7.4) for $2 \mathrm{~h}$ and washed with cacodylate buffer. Post-fixing was carried out in $1 \%$ osmium tetroxide and $1.5 \%$ potassium ferrocyanide for $1 \mathrm{~h}$. After dehydration with $50-100 \%$ alcohol, the cells were embedded in Poly/Bed 812 resin (Pelco, Redding, CA, USA), polymerized and observed under electron microscope (EM 902A, Zeiss).

\section{Isobologram analysis}

To determine the effect of combination of bortezomib and nutlin-3 on MDA-MB 435S, DLD-1, HeLa and T98G cells, dose-dependent effects were determined for each compound and for one compound with fixed concentrations of another. The interaction of bortezomib and nutlin-3 was quantified by determining the combination index (CI), in accordance with the following classic isobologram (Chou TC \& Talalay P., 1984). The equation for the isobologram is shown as $\mathrm{CI}=(\mathrm{D})_{1} /(\mathrm{Dx})_{1}+(\mathrm{D})_{2} /(\mathrm{Dx})_{2}$, where $(\mathrm{Dx})_{1}$ and $(\mathrm{Dx})_{2}$ indicate the individual dose of bortezomib and nutlin-3 required to produce an effect, and $(D)_{1}$ and $(D)_{2}$ are the doses of bortezomib and nutlin-3, respectively, in combination that produce the same effect. From this analysis, the combined effects of the two drugs can be summarized as follows: $\mathrm{CI}<1$ indicates synergism; $\mathrm{CI}=1$ indicates summation (additive and zero interaction); and $\mathrm{CI}>1$ indicates antagonism.

\section{Statistical analysis}

Statistical analysis was performed with GraphPad Prism (GraphPad Software, Sandiego, CA, USA) was used. Unless otherwise specified, each experiment was repeated at least three times. Normality of data was assessed by Kolmogorov-Smirnov testes and equal variance using Bartlett's test. For normal distribution, statistical differences were determined using an analysis of variance (ANOVA) followed by Bonferroni multiple comparison test. If the data were not normally distributed, Kruskal-Wallis test was performed followed by Dunn's test. $P<0.05$ was considered statistically significant.

\section{RESULTS}

Bortezomib and nutlin-3 synergistically induce cell death accompanied by vacuolation in various $p 53$-defective cancer cell lines

Bortezomib and nutlin-3 were previously shown to synergistically induce apoptosis in various cell types, including multiple myeloma cells ${ }^{29,30}$ and MCL cells. ${ }^{31}$ In the present study, we tested the effects of the combination of bortezomib and nutlin- 3 on various solid tumor cell lines. The cancer cells used in this study commonly harbored functionally defective p53: MDA-MB 435S breast cancer cells have G266E-mutant p53, ${ }^{32}$ T98G glioma cells have M237I-mutant p53,33 DLD-1 colon cancer cells have S241P mutant p53, ${ }^{34}$ and HeLa cervical cancer cells have non-functional p53 due to the expression of E6. ${ }^{35,36}$ Thus, our investigations focused on the p53-independent sensitizing activities of nutlin-3. While treatment with nutlin-3 up to $30 \mu \mathrm{M}$ was not cytotoxic to these cancer cells, MDA-MB 435S, T98G, DLD-1 and HeLa cells were resistant to bortezomib up to 10, 6, 40 and $50 \mathrm{~nm}$, respectively (Figure 1a). In contrast, nutlin-3 commonly and dose-dependently enhanced cell death when combined with subtoxic doses of bortezomib. Particularly, in HeLa cells, which were very resistant to bortezomib ( $\left.\mathrm{IC}_{50}>8.0 \mu \mathrm{M}\right), 10 \mathrm{nM}$ bortezomib was sufficient to kill $50 \%$ of these cells when combined with $30 \mu \mathrm{M}$ nutlin-3. Isobologram analysis showed that bortezomib and nutlin-3 synergistically induced cell death in all of the tested cancer cell lines (Figure 1b). Taken together, these results indicate that nutlin-3 effectively overcomes the bortezomib resistance of the tested p53-defective cancer cell lines. To confirm that nutlin-3 sensitizes cells to bortezomib-mediated cell death in a p53-independent manner, we additionally examined two HCT116 isogenic cell lines that differ in their p53 status: HCT116 p53 wild-type (WT) cells and HCT116 p53-null (p53-/- $)$ cells. Interestingly, nutlin-3 alone demonstrated dose-dependent cytotoxicity in HCT116 WT cells, but this was almost completely blocked by pretreatment with pifithrin- $\alpha$, which is a specific inhibitor of p53-mediated transcriptional activity (Figure 1c). ${ }^{37}$ In contrast, treatment with $20 \mu \mathrm{M}$ nutlin-3 alone did not induce cell death in HCT116 
p53-/- cells, indicating that the cytotoxic effect of nutlin-3 alone depends on p53. Bortezomib alone ( $5 \mathrm{~nm})$ did not induce

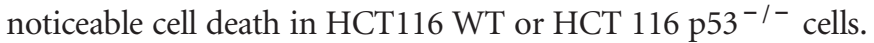
Co-treatment of HCT116 WT cells with bortezomib and nultin-3 slightly increased the observed nutlin-3-induced cytotoxicity, and this effect was partially alleviated by

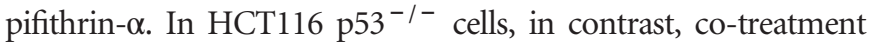
with bortezomib and nutlin-3 (bortezomib/nutlin-3) effectively a
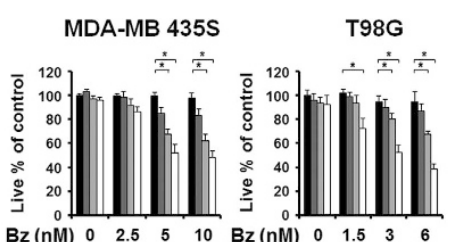

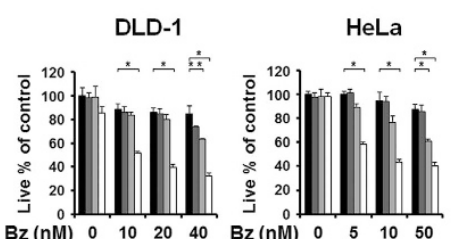

C

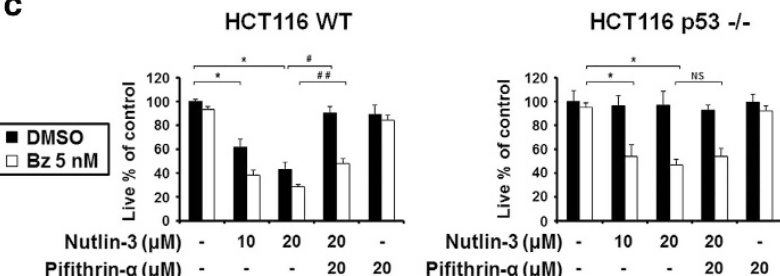

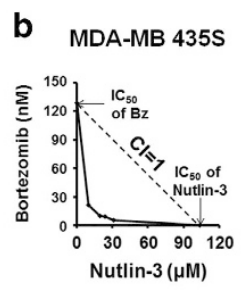

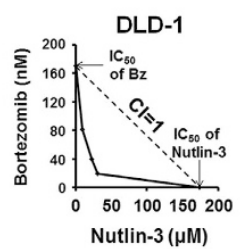

d

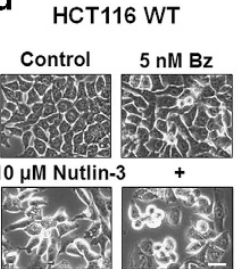

T98G
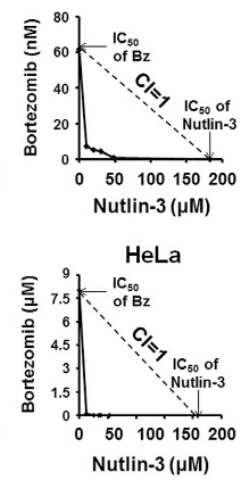

HCT116 p53 -/-

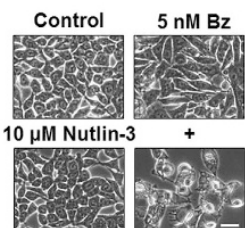

e
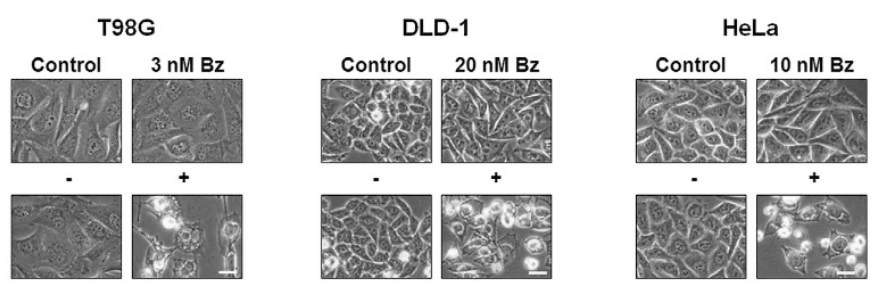

f

MDA-MB 435S
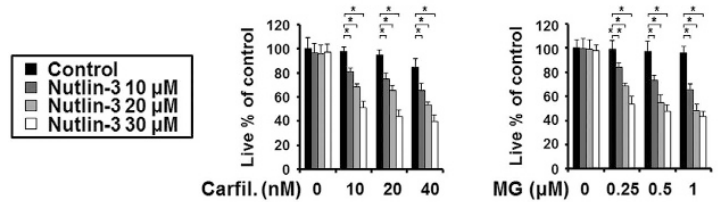

h

MDA-MB 435S
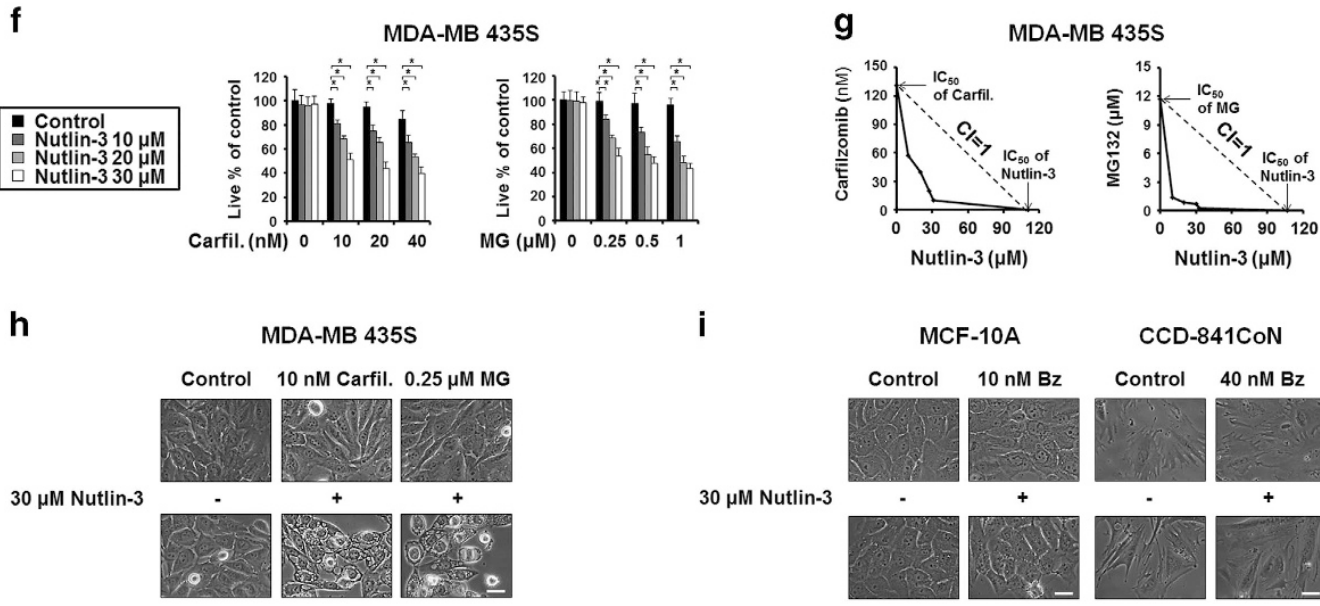

j
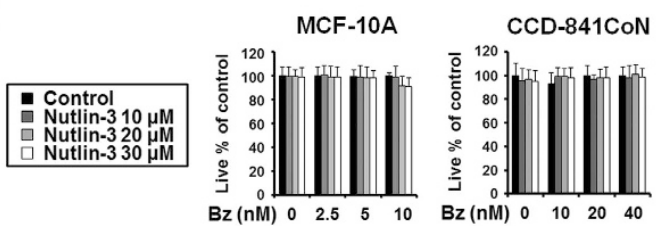

i

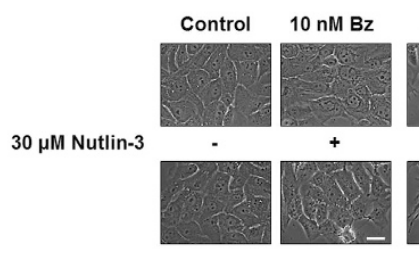

CCD-841CoN

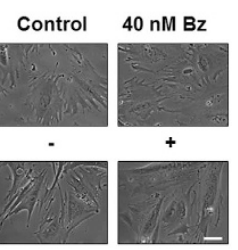


induced cell death, regardless of pifithrin- $\alpha$ pretreatment (Figure 1c). Phase-contrast microscopy confirmed that $5 \mathrm{~nm}$ bortezomib alone had no effect on HCT116 WT and HCT116 p53 $3^{-1-}$ cells (Figure 1d). Treatment with $20 \mu \mathrm{m}$ nultin-3 triggered cellular detachment due to death in HCT116 WT cells, but not in HCT116 $\mathrm{p}^{-/-}$cells. Bortezomib/ nutlin-3 markedly induced cell death in both cell lines, but notable vacuolation was seen in HCT116 $\mathrm{p}^{-/-}$cells. Taken together, these results indicate that nutlin-3 can overcome the bortezomib resistance of the tested cancer cell lines via a novel mechanism that does not require $\mathrm{p} 53$.

Next, we examined whether bortezomib/nutlin-3 induces cell death accompanied by vacuolation in other p53-defective cancer cell lines. Treatment with $30 \mu \mathrm{m}$ nutlin-3 alone did not noticeably alter the cellular morphologies of MDA-MB 435S, T98G, DLD-1 or HeLa cells (Figure 1e). Treatment with subtoxic doses of bortezomib (5 $\mathrm{nM}$ in MDA-MB $435 \mathrm{~S}$ cell, $3 \mathrm{~nm}$ in T98G, $20 \mathrm{~nm}$ DLD-1 and $10 \mathrm{~nm}$ in HeLa cells) also did not noticeably affect cellular morphology. In contrast, bortezomib/nutlin-3 commonly induced extensive cytoplasmic vacuolation and cell death in these cancer cells. Next, we tested whether nutlin-3 could sensitize cancer cells to the effects of other proteasome inhibitors. We found that treatment with nutlin-3 and subtoxic doses of carfilzomib or MG132 also dose-dependently and synergistically induced cell death in MDA-MB 435S cells (Figure if and g). Similar to bortezomib/ nutlin-3, both carfilzomib/nutlin-3 and MG132/nutlin-3, but not $10 \mathrm{~nm}$ carfilzomib or $0.25 \mu \mathrm{M}$ MG132 alone, induced marked vacuolation (Figure 1h). Thus, these results suggest that nutlin-3 may effectively sensitize cancer cells to death when combined with various proteasome inhibitors. To further examine the effect of bortezomib/nutlin-3 on normal cells, we employed MCF-10A breast cells and CCD-841CoN colon epithelial cells as counterpart cells for breast cancer cells and colon cancer cells, respectively. We found that both MCF-10A and CCD-841CoN cells were resistant to the indicated concentrations of bortezomib/nutlin-3 (Figure 1i). In addition, we could not observe notable cytoplasmic vacuolation (Figure 1i) or cell death (Figure 1j) in bortezomib/nutlin-3-treated MCF-10A and CCD-841CoN cells. These results suggest that bortezomib/nutlin-3 treatment may be preferentially cytotoxic to cancer cells (at least those of the breast and colon) over their normal counterpart cells.

To examine the cell death mode induced by bortezomib/ nutlin-3, we first tested the effects of the apoptosis inhibitor, z-VAD-fmk. Bortezomib/nutlin-3-induced cytoplasmic vacuolation and cell death were not altered by z-VAD-fmk in any of the tested cancer cell lines (Supplementary Figure 1a and b). However, when we assessed cell viability using calcein-AM and EthD-1 and employing TRAIL as a representative apoptotic inducer, we found that $\mathrm{z}-\mathrm{VAD}$-fmk pretreatment effectively inhibited TRAIL-induced cell death in MDA-MB $435 \mathrm{~S}$ cells (Supplementary Figure 1c). Flow cytometry of annexin V/PI-stained cells showed that treatment of MDA-MB 435S cells with TRAIL for $48 \mathrm{~h}$ increased both the annexin $\mathrm{V}(+) / \mathrm{PI}$ $(+)$ and annexin V (-)/PI $(+)$ cell populations, indicating that TRAIL may induce both late apoptosis and necrosis. In contrast, we did not notably detect annexin $\mathrm{V}(+)$ or PI $(+)$ cells in cultures treated with bortezomib/nutlin-3 for $24 \mathrm{~h}$ (Supplementary Figure 1d). Taken together, these results suggest that apoptosis may not be a major cell death mode in the anti-cancer effect of bortezomib/nutlin-3. Moreover, the abilities of bortezomib/nutlin-3 to induce cytoplasmic vacuolation and cell death in the tested cancer cells were not affected by necrostatin-1 (an inhibitor of necroptosis), 3-MA, or bafilomycin A (an inhibitor of autophagy) (Supplementary Figure 1a and b). Collectively, our findings suggest that an alternative cell death mode that does not involve apoptosis, necroptosis, or autophagic cell death may be critically involved in the bortezomib/nutlin-3-indcued killing of breast cancer cells.

\section{Bortezomib/nutlin-3 induces paraptosis in various p53-defective cancer cell lines}

We recently showed that curcumin or celastrol can induce paraptosis, and that proteasome inhibition plays a critical role in this cell death. ${ }^{18,20}$ Here we tested whether the vacuolation induced by bortezomib/nutlin-3 is associated with paraptotic changes. For this purpose, we employed MDA-MB 435S sublines transfected with the YFP-ER or YFP-Mito plasmids,

Figure 1 Bortezomib and nutlin-3 synergistically induce the cell death accompanied by cytoplasmic vacuolation in various cancer cells. $(\mathbf{a}, \mathbf{j})$ Cells were treated with the indicated concentrations of bortezomib and/or nutlin-3 for $24 \mathrm{~h}$ and cellular viability was assessed using calcein-AM and EthD-1. The percentage of live cells was normalized to that of untreated control cells (100\%). Data represent the means \pm s.d. One-way ANOVA and Bonferroni's post hoc test. ${ }^{*} P<0.005,{ }^{*} P<0.01$ vs untreated cells. The experiment was repeated at least three times with similar results. (b) Isoboles for the combination of bortezomib and nutlin-3, which were isoeffective (IC 50 ) for inhibition of cell viability, are shown. (c) Two HCT116 isogenic cell lines were treated with bortezomib and/or nutlin-3 at the indicated concentration in the absence or presence of $20 \mu \mathrm{m}$ pifithrin- $\alpha$ for $24 \mathrm{~h}$. Cellular viability was assessed using calcein-AM and EthD-1. Data represent the means \pm s.d. Kruskal-Wallis test was performed followed by Dunn's test. ${ }^{*} P<0.005$ vs untreated cells, $P<0.005$, \#\# $P<0.01$ vs cells treated with $20 \mu \mathrm{m}$ nutlin-3, NS, not significant. (d, e, i) Cells were treated with the indicated concentration of bortezomib and/or nutlin-3 for $24 \mathrm{~h}$ and observed under the phase-contrast microscope. Scale bars, $10 \mu \mathrm{m}$. (f) MDA-MB 435S cells were treated with the indicated concentrations of carfilzomib and/or nutlin-3 or MG132 and/or nutlin-3 for $24 \mathrm{~h}$ and cellular viability was assessed using calcein$\mathrm{AM}$ and EthD-1. Data represent the means \pm s.d. One-way ANOVA and Bonferroni's post hoc test. ${ }^{*} P<0.005,{ }^{* *} P<0.01$ vs untreated cells. (g) Isoboles for the combination of carfilzomib and nutlin-3 or isoboles for the combination of MG132 and nutlin-3, which were isoeffective $\left(\mathrm{IC}_{50}\right)$ for inhibition of cell viability, are shown. (h) MDA-MB 435S cells were treated with the indicated concentrations of carfilzomib and/or nutlin-3 or MG132 and/or nutlin-3 for $24 \mathrm{~h}$ and observed under the phase-contrast microscope. Scale bars, $10 \mu \mathrm{m}$. 
which label the ER or mitochondria, respectively (YFP-ER cells and YFP-Mito cells, respectively). Fluorescence microscopy of YFP-ER cells showed that the ER structures of reticular shape were not markedly affected by $5 \mathrm{~nm}$ bortezomib or $30 \mu \mathrm{M}$ nutlin-3 alone (Figure 2a). In contrast, cells treated with bortezomib/nutlin-3 for $8 \mathrm{~h}$ exhibited fluorescence within numerous cytosolic vacuoles, suggesting that these vacuoles were derived from the ER. As the incubation time increased,

a

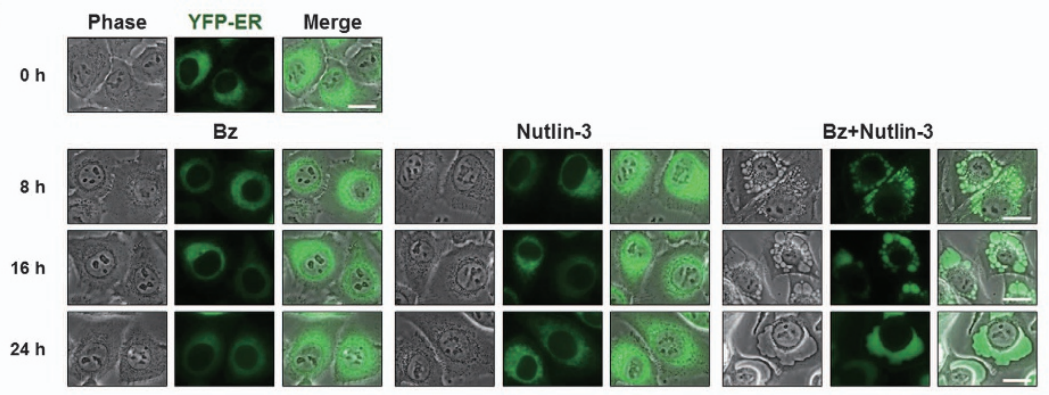

b

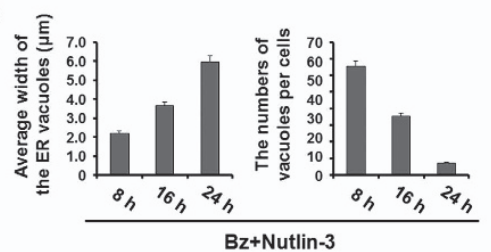

C
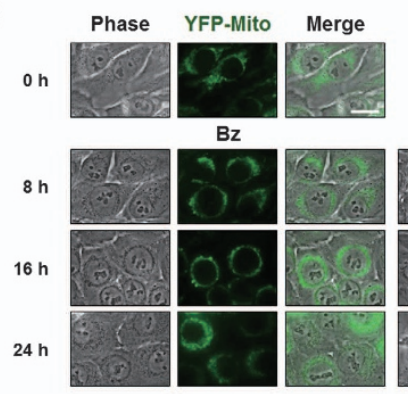

Nutlin-3
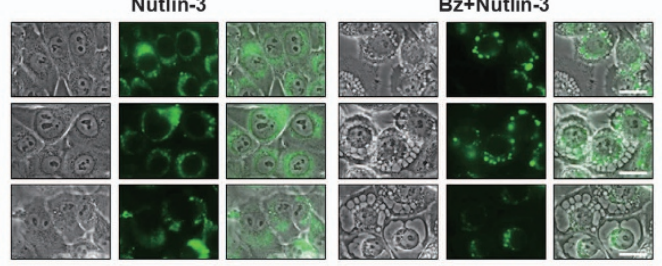

d
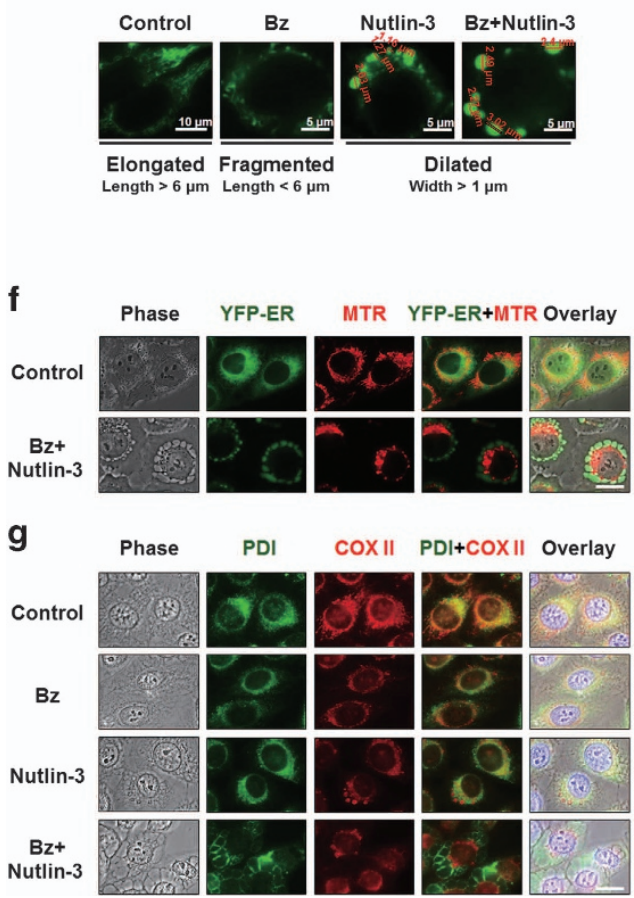

e

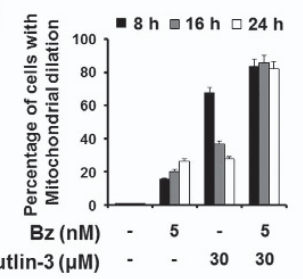

h

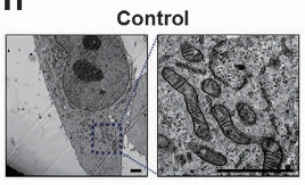

Bz
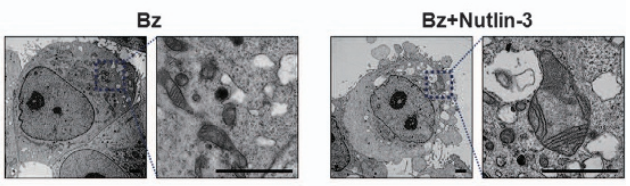

Bz+Nutlin-3

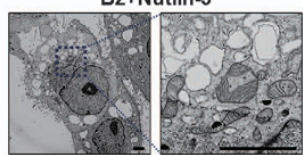


these ER-derived vacuoles increased in size and decreased in number (Figure 2b), suggesting that the swollen ER underwent progressive fusion events. The mitochondria of untreated YFPMito cells exhibited filamentous and elongated morphologies, whereas those of the corresponding $5 \mathrm{~nm}$ bortezomib-treated cells exhibited mitochondrial fragmentation (Figure 2c). Interestingly, treatment with nutlin-3 alone induced mitochondrial dilation (to an average width $>1 \mu \mathrm{m}$ ) from 8 to $16 \mathrm{~h}$ post treatment (Figure $2 \mathrm{c}$ and $\mathrm{d}$ ). However, the mitochondriaderived vacuoles were slightly reduced in size after $24 \mathrm{~h}$ of treatment of cells with nutlin-3 alone, suggesting that mitochondria might be transiently dilated and then undergo fission in these cells. Co-treatment with bortezomib/nutlin-3 induced a marked dilation of mitochondria around the nuclei by $8 \mathrm{~h}$ post treatment; notably, these mitochondria-derived vacuoles were much bigger than those induced by nutlin-3 alone (Figure $2 \mathrm{c}$ and $\mathrm{d}$ ). The fluorescence intensity of the mitochondria-derived vacuoles was somewhat weakened at $24 \mathrm{~h}$ post treatment with bortezomib/nutlin-3 (Figure 2c). When we assessed the percentages of cells with mitochondrial dilation, our findings indicated that bortezomib/nultin-3 induced a mitochondrial dilation that began at $8 \mathrm{~h}$ and continued thereafter, whereas treatment with nutlin- 3 alone triggered a transient increase of mitochondrial dilation that peaked at $8 \mathrm{~h}$ post treatment (Figure 2e). Bortezomib/nutlin-3induced dilation of both the ER and mitochondria was also observed by the fluorescence microscopy of YFP-ER cells following by staining with MTR (Figure 2f). We further performed immunocytochemistry of COX II, a protein localized in the inner mitochondrial membrane, and PDI, an ER-resident protein. In MDA-MB $435 \mathrm{~S}$ cells treated with bortezomib/nutlin-3, COX II expression was detected as a small ring shape at the perinuclear area, while PDI expression was observed as a larger ring shape at the cellular periphery (Figure 2g). Similar results were obtained in T98G, DLD-1 and HeLa cells treated with bortezomib/nutlin-3 (Supplementary Figure 2), suggesting that bortezomib/nutlin-3 induces dilation of the ER and mitochondria in various cancer cell lines. Electron microscopy revealed that treatment of MDA-MB $435 \mathrm{~S}$ cells with $5 \mathrm{~nm}$ bortezomib for $8 \mathrm{~h}$ induced mitochondrial fragmentation with disruption of cristae structures and a very slight swelling of the ER (Figure $2 \mathrm{~h}$ ). In contrast, treatment with $30 \mu \mathrm{m}$ nutlin-3 alone induced a mitochondrial swelling in which the cristae structures were pushed toward the periphery, and no obvious alteration of the ER structure was seen. Interestingly, in cells treated with bortezomib/nutlin-3, fusion among the swollen ER and formation of megamitochondria (MG) were noted. Thus, bortezomib/nutlin-3 appears to induce the morphological features of paraptosis in various p53-defective cancer cells.

Since paraptosis is known to require active protein synthesis, we further tested the effect of the protein synthesis inhibitor, $\mathrm{CHX}^{38}$ We found that $\mathrm{CHX}$ pretreatment very effectively blocked the cell death induced by bortezomib/nutlin-3 in MDA-MB 435S, T98G, DLD-1 and HeLa cells (Figure 3a). Phase-contrast microscopy showed that $\mathrm{CHX}$ pretreatment dose-dependently inhibited bortezomib/nutlin-3-induced cellular vacuolation and cell death in MDA-MB $435 \mathrm{~S}$ cells (Figure $3 \mathrm{a}$ and $\mathrm{b}$ ). Furthermore, CHX pretreatment effectively inhibited the vacuolation and cell death also in T98G, DLD-1, and HeLa cells treated with bortezomib/nutlin-3 (Figure 3a and c). Furthermore, fluorescence microscopy of MTR-stained YFP-ER cells showed that CHX pretreatment completely blocked the bortezomib/nutlin-3-induced dilation of the ER and mitochondria (Figure 3d). Collectively, these results indicate that bortezomib/nutlin-3 induces paraptosis in various cancer cells independently of p53.

\section{CHOP induction critically contributes to ER dilation during bortezomib/nultin-3-induced paraptosis}

To investigate how nutlin-3 enhances bortezomib sensitivity in the tested cancer cell lines, we first examined whether nutlin-3 affects bortezomib-mediated proteasome inhibition. We found that the bortezomib-induced accumulation of poly-ubiquitinated proteins was further potentiated by nutlin-3 co-treatment in MDA-MB 435S, T98G, DLD-1 and HeLa cells (Figure 4a). In addition, combined treatment commonly and markedly increased the protein levels of CHOP, a bona fide ER stress marker, ${ }^{39}$ compared to either bortezomib or nutlin-3 alone. A time-course experiment showed that bortezomib/ nutlin-3 treatment progressively increased the protein levels of both poly-ubiquitinated proteins and CHOP (Figure 4b). These results suggest that co-treatment with nutlin-3 aggravates the bortezomib-mediated impairment of proteasomal activity

Figure 2 Vacuolation induced by bortezomib/nutlin-3 is derived from the dilation of both the ER and mitochondria. (a, c) YFP-ER/435S cells expressing the fluorescence selectively in the ER (a) or YFP-Mito/435S cells expressing the fluorescence selectively in mitochondria (c), were treated with $5 \mathrm{~nm}$ bortezomib and/or $30 \mu \mathrm{m}$ nutlin-3 for the indicated time points and observed under the fluorescent and phase-contrast microscope. Scale bars, $20 \mu \mathrm{m}$. (b) The average widths of the vacuoles originated from the ER and the numbers of the vacuoles per cells were measured in YFP-ER cells treated with bortezomib and/or nutlin-3 for the indicated time points using AxioVision Rel. 4.8 software (Zeiss). (d) Mitochondrial morphology of 'elongated', 'fragmented' and 'dilated' was defined as the mitochondria with the length longer than $6 \mu \mathrm{m}$, those with shorter than $6 \mu \mathrm{m}$, and those with widths longer than $1 \mu \mathrm{m}$. Representative mitochondrial morphologies of YFP-Mito/435S cells treated as indicated are shown. (e) YFP-Mito/435S cells with mitochondrial dilation were quantified following treatment with $5 \mathrm{~nm}$ bortezomib and/or $30 \mu \mathrm{m}$ nutlin-3 for the indicated time points. (f) YFP-ER/435S cells treated with $5 \mathrm{~nm}$ bortezomib and $30 \mu \mathrm{m}$ nutlin-3 for $8 \mathrm{~h}$ were stained with $100 \mathrm{~nm}$ MitoTracker-Red (MTR) and observed under the phase-contrast and fluorescence microscope. Scale bar, $20 \mu \mathrm{m}$. (g) MDA-MB 435S cells were treated with $5 \mathrm{~nm}$ bortezomib and/or $30 \mu \mathrm{m}$ nutlin-3 for $16 \mathrm{~h}$, fixed, and subjected to the immunocytochemistry of PDI and COX II. Scale bar, $20 \mu \mathrm{m}$. (h) MDA-MB 435S cells were treated with 5 nM bortezomib and/or $30 \mu \mathrm{m}$ nutlin-3 for $8 \mathrm{~h}$, fixed, and subjected to electron microscopy. Scale bars, $0.5 \mu \mathrm{m}$. 
a

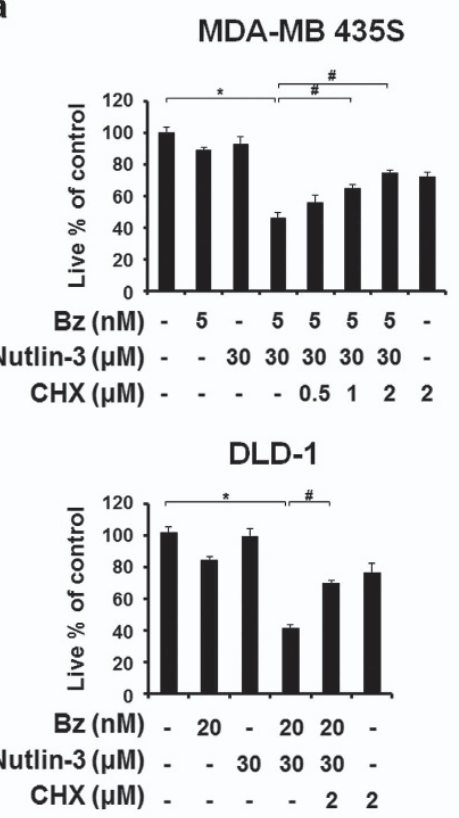

T98G

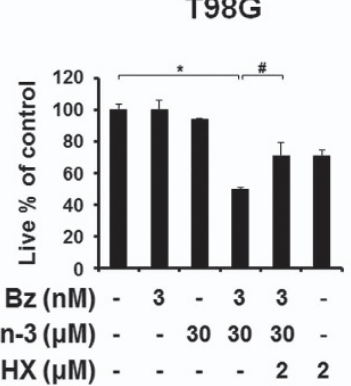

HeLa

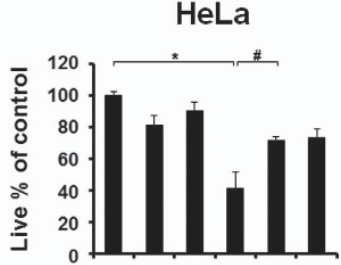

$\mathrm{Bz}(\mathrm{nM})$ - 10 - 1010 Nutlin-3 ( $\mu \mathrm{M})$ - $\quad-\begin{array}{lllll}30 & 30 & 30\end{array}$ $\mathrm{CHX}(\mu \mathrm{M})$ - - - -22 b

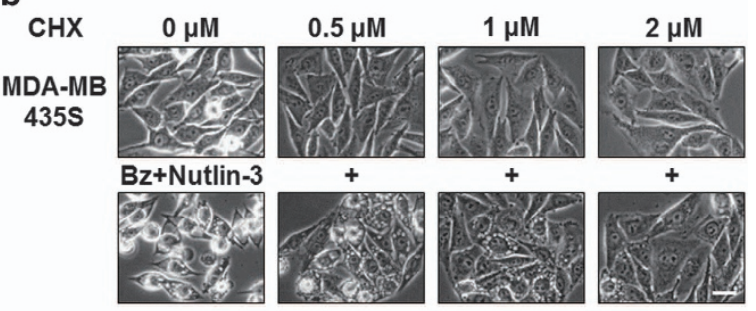

c

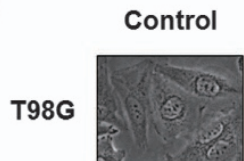

CHX

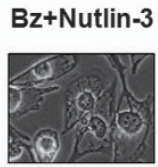

$\mathrm{CHX}+$ Bz+Nutlin-3
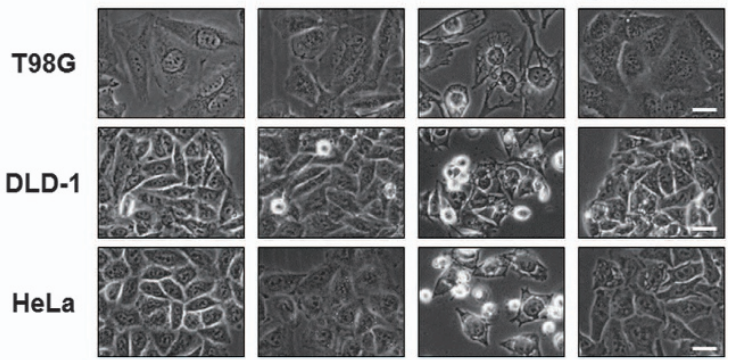
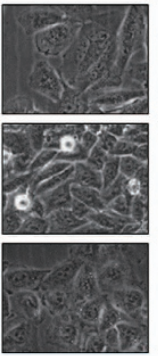
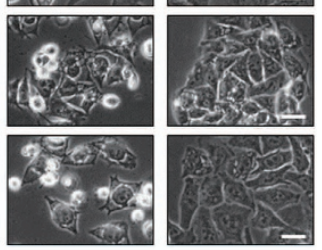

d
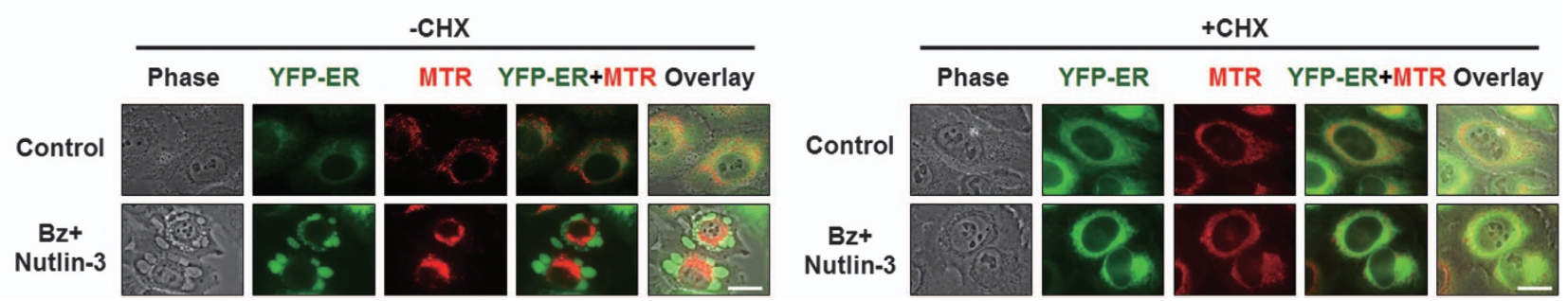

Figure $3 \mathrm{CHX}$ pretreatment effectively blocks the vacuolation and cell death induced by bortezomib/nutlin-3. (a) The respective cells pretreated with or without $\mathrm{CHX}$ were further treated with the indicated concentrations of bortezomib plus nutlin-3 for $24 \mathrm{~h}$. Cell viability was assessed using calcein-AM and EthD-1. Data represent the means \pm s.d. One-way ANOVA and Bonferroni's post hoc test. ${ }^{*} P<0.005$ vs untreated cells; ${ }^{\#} P<0.005$ vs cells treated with bortezomib/nutlin-3. The experiment was repeated at least three times with similar results. (b) MDA-MB $435 \mathrm{~S}$ cells pretreated with $\mathrm{CHX}$ at the indicated concentrations were further treated with $5 \mathrm{~nm}$ bortezomib plus $30 \mu \mathrm{m}$ nutlin-3 for $24 \mathrm{~h}$ and observed under the phase-contrast microscope. Scale bar, $10 \mu \mathrm{m}$. (c) T98G cells treated with $3 \mathrm{~nm}$ bortezomib plus $30 \mu \mathrm{m}$ nutlin-3, DLD-1 cells treated with $20 \mathrm{~nm}$ bortezomib plus $30 \mu \mathrm{m}$ nutlin-3 and HeLa cells treated with $10 \mathrm{~nm}$ bortezomib plus $30 \mu \mathrm{m}$ nutlin-3 for $16 \mathrm{~h}$ in the absence or presence of $\mathrm{CHX}$ were observed under the phase-contrast microscope. Scale bars, $10 \mu \mathrm{m}$. (d) YFP-ER/ $435 \mathrm{~S}$ cells were pre-treated with or without $2 \mu \mathrm{m} \mathrm{CHX}$ and further treated with $5 \mathrm{~nm}$ bortezomib and $30 \mu \mathrm{m}$ nutlin-3 for $8 \mathrm{~h}$. Treated cells were stained with $100 \mathrm{~nm} \mathrm{MTR}$ and observed under the phase-contrast and fluorescence microscope. Scale bars, $20 \mu \mathrm{m}$.

and subsequent ER stress. Accordingly, we investigated the functional significance of $\mathrm{CHOP}$ induction for the cell death induced by bortezomib/nutlin-3. When we incubated MDA-MB 4355 cells with lentiviruses containing non-targeting shRNA (shNT) or CHOP-targeting shRNA (shCHOP) and further treated the cells with bortezomib/nutlin-3, we found that both cell death and vacuolation were significantly attenuated by CHOP knockdown (Figure 4c and d). Moreover, immunocytochemical analysis of PDI and COX II showed that CHOP knockdown remarkably inhibited the dilation of the ER induced by bortezomib/nutlin-3 (Figure 4e), but did not affect the mitochondrial dilation induced by bortezomib/nutlin-3 or nutlin-3 alone. Taken together, these results suggest that CHOP plays a critical role in bortezomib/nutlin-3-induced ER dilation, contributing to the paraptosis induced by this co-treatment.
Cytosolic $\mathrm{Ca}^{2+}$ plays an important role in the ER-derived vacuolation and cell death induced by bortezomib/nutlin-3 Since bortezomib/nutlin-3 induced marked structural changes in the ER, a major reservoir of intracellular $\mathrm{Ca}^{2+}$, we next examined whether this co-treatment affected intracellular $\mathrm{Ca}^{2+}$ homeostasis. Flow cytometry of cells exposed to the cell-permeable calcium indicator dye, Fluo-3, demonstrated that bortezomib/nutlin-3 time-dependently increased intracellular $\mathrm{Ca}^{2+}$ levels in MDA-MB $435 \mathrm{~S}$ cells, whereas treatment with bortezomib or nutlin-3 alone had no such effect (Figure 5a). To investigate the functional significance of the increased $\mathrm{Ca}^{2+}$ levels observed during bortezomib/nutlin-3indcued cell death, we tested the effect of the extracellular $\mathrm{Ca}^{2+}$ scavenger, BAPTA, or the intracellular $\mathrm{Ca}^{2+}$ scavenger, BAPTA-AM. We found that both BAPTA and BAPTA-AM dose-dependently inhibited the cell death induced by 


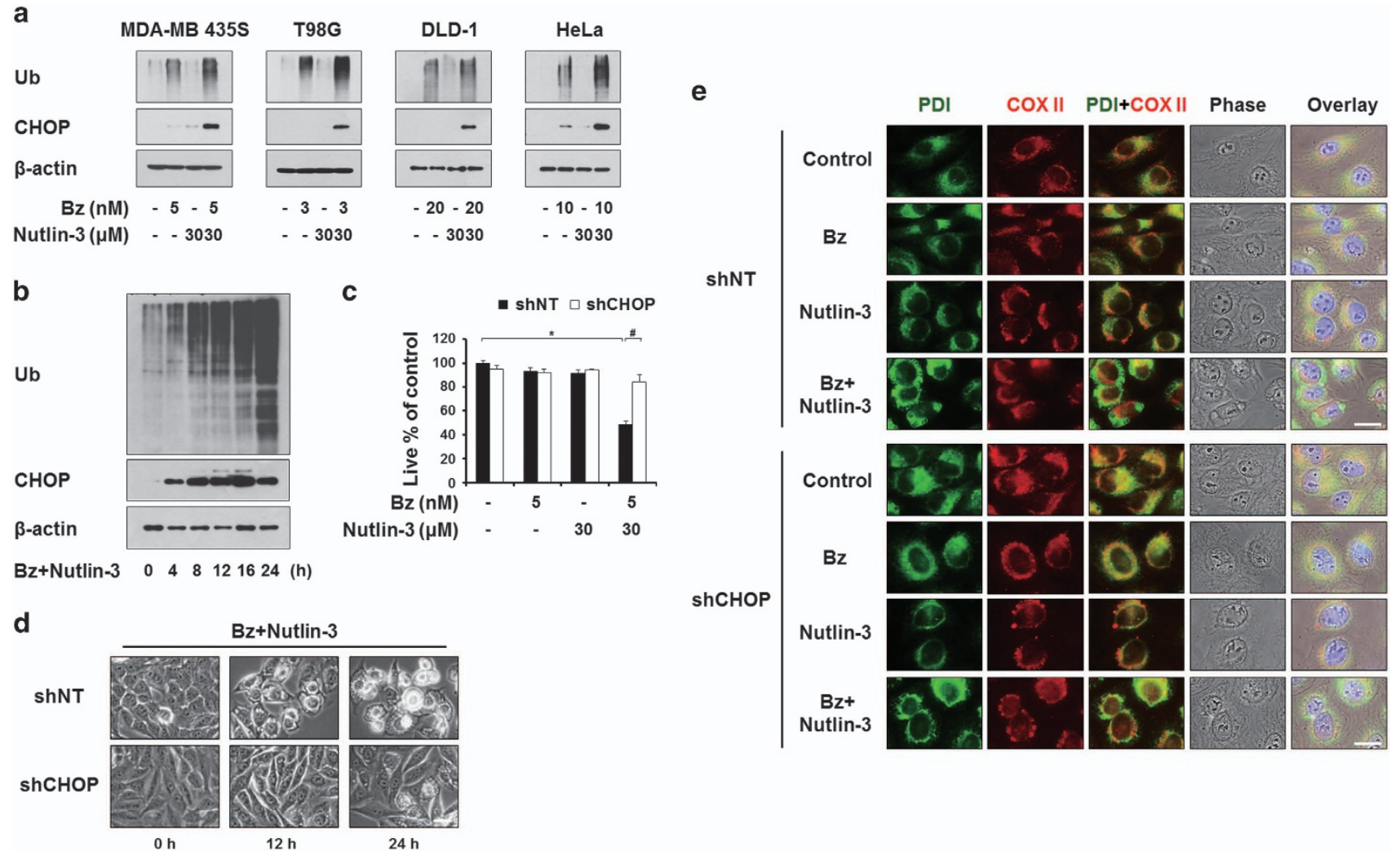

Figure $4 \mathrm{CHOP}$ induction critically contributes to the dilation of the ER and subsequent cell death by bortezomib/nultin-3. (a) Cell extracts were prepared from MDA-MB 435S cells treated with the indicated concentrations of bortezomib and/or nutlin-3 for $8 \mathrm{~h}$ and western blotting of the proteins associated with ER stress was performed. $\beta$-actin was used as a loading control in western blots. (b) Cell extracts were prepared from MDA-MB 435S cells treated with $5 \mathrm{~nm}$ bortezomib plus $30 \mu \mathrm{m}$ nutlin-3 for indicated time points and western blotting of ubiquitin and CHOP was performed. $\beta$-Actin was used as a loading control in western blots. (c-e) MDA-MB 435S cells were infected with the lentivirus containing non-targeting (NT) shRNA or a CHOP-targeting shRNA (CHOP shRNA) for $24 \mathrm{~h}$. Infected cells were treated with $5 \mathrm{~nm}$ bortezomib plus $30 \mu \mathrm{m}$ nutlin-3 for $24 \mathrm{~h}$ (c, d) or for $16 \mathrm{~h}$ (e). (c) Cell viability was assessed using calcein-AM and EthD-1. The percentage of live cells was normalized to that of cells transfected with shNT without treatment (100\%). Data represent the means \pm s.d. One-way ANOVA and Bonferroni's post hoc test. ${ }^{*} P<0.005$ vs cells transfected with shNT without treatment, ${ }^{\#} P<0.005$ vs cells transfected with shNT and further treated with bortezomib/nutlin-3. The experiment was repeated at least three times with similar results. (d) Treated cells as indicated were observed under the phase-contrast microscope. Scale bar, $10 \mu \mathrm{m}$. (e) Treated cells as indicated were fixed and subjected for immunocytochemistry of PDI and COX II. Scale bars, $20 \mu \mathrm{m}$.

bortezomib/nutlin-3, with BAPTA-AM demonstrating a stronger inhibitory effect (Figure 5b). Co-treatment with BAPTA and BAPTA-AM more potently blocked the cell death induced by bortezomib/nutlin-3 compared to either single treatment. Furthermore, pretreatment with BAPTA plus BAPTA-AM effectively blocked the vacuolation induced by bortezomib/nutlin-3, and was associated with marked scavenging of the increased $\mathrm{Ca}^{2+}$ (Figure 5c). When we further examined the morphologies of the ER and mitochondria employing YFP-ER cells and MTR, dilation of the ER, but not mitochondrial dilation, was effectively blocked by pretreatment with BAPTA plus BAPTA-AM (Figure 5d). Collectively, these results suggest that disruption of intracellular $\mathrm{Ca}^{2+}$ homeostasis due (at least in part) to an influx of extracellular $\mathrm{Ca}^{2+}$ may critically contribute to the ER dilation and subsequent cell death induced by bortezomib/ nutlin-3.
Mitochondrial $\mathrm{Ca}^{2+}$ overload contributes to the cell death induced by bortezomib/nutlin-3 but may not directly cause the observed mitochondrial dilation

When cytosolic $\mathrm{Ca}^{2+}$ levels are high, $\mathrm{Ca}^{2+}$ can enter mitochondria though the MCU. ${ }^{40,41}$ Therefore, we tested whether bortezomib/nutlin-3 increases mitochondrial $\mathrm{Ca}^{2+}$ levels and, if so, whether this effect is important for the paraptosis induced by the combined treatment. We measured changes in mitochondrial $\mathrm{Ca}^{2+}$ levels by flow cytometry using Rhod-2 (a cell-permeable mitochondrial calcium-indicator dye), and found that treatment of MDA-MB $435 \mathrm{~S}$ cells with bortezomib/ nutlin-3, but not either agent alone, increased the mitochondrial calcium levels to a peak at $12 \mathrm{~h}$ post treatment (Figure 6a). Since MCU-mediated mitochondrial $\mathrm{Ca}^{2+}$ uptake is known to be inhibited by Ru360, ${ }^{41}$ we next investigated the effect of Ru360 on the cell death by bortezomib/nutlin-3. We found that Ru360 pretreatment significantly inhibited this parameter 
a

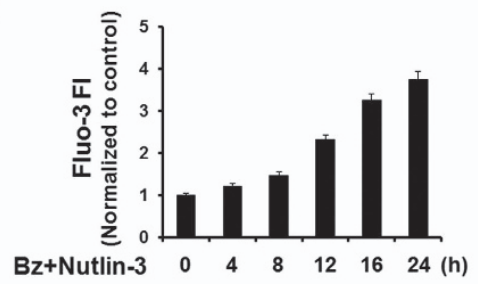

b

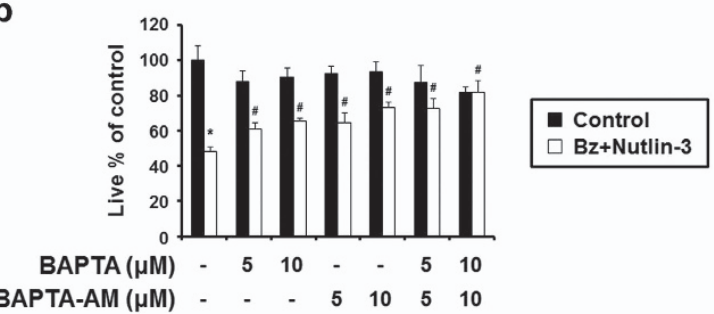

C
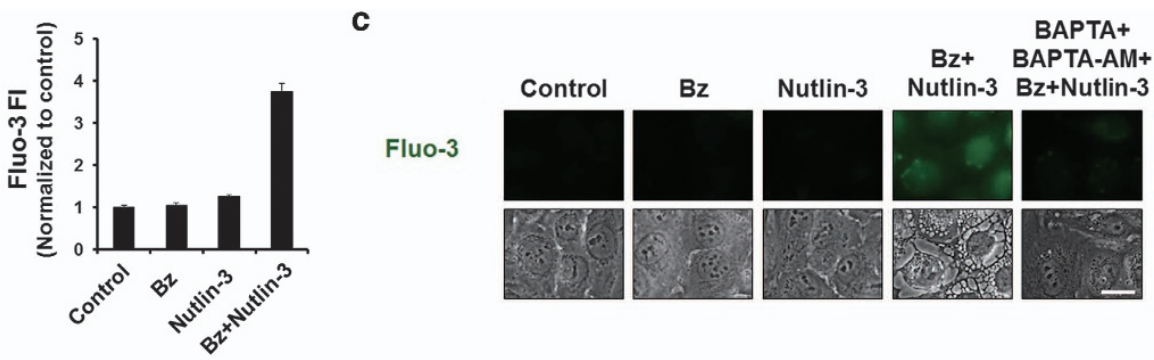

d

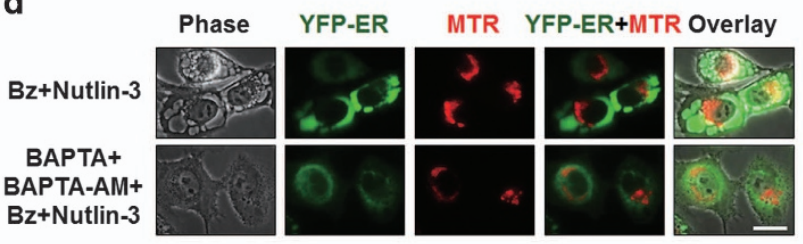

Figure 5 Disruption of intracellular $\mathrm{Ca}^{2+}$ homeostasis critically contributes to the dilation of the ER and subsequent cell death by bortezomib/nultin-3. (a) MDA-MB 435S cells treated with $5 \mathrm{~nm}$ bortezomib plus $30 \mu \mathrm{m}$ nutlin-3 for the indicated time points were subjected to flow cytometry using $1 \mu \mathrm{M}$ Fluo-3 and changes in $\mathrm{Ca}^{2+}$ levels were compared among the treatment groups. (b) MDA-MB 435S cells were pretreated with BAPTA, BAPTA-AM or BAPTA plus BAPTA-AM at the indicated concentrations and further treated with 5 nM bortezomib plus $30 \mu \mathrm{m}$ nutlin-3 for $24 \mathrm{~h}$. Cell viability was measured using calcein-AM and EthD-1. Data represent the means \pm s.d. One-way ANOVA and Bonferroni's post hoc test. ${ }^{*} P<0.005$ vs untreated cells, ${ }^{\#} P<0.005$ vs bortezomib/nutlin-3-treated group. The experiment was repeated at least three times with similar results. (c) MDA-MB 435S cells were pretreated with $10 \mu \mathrm{m}$ BAPTA, $10 \mu \mathrm{m}$ BAPTA-AM or $10 \mu \mathrm{m}$ BAPTA plus $10 \mu \mathrm{m}$ BAPTA-AM and further treated with $5 \mathrm{~nm}$ bortezomib plus $30 \mu \mathrm{m}$ nutlin-3 for $16 \mathrm{~h}$. Treated cells were stained with $1 \mu \mathrm{m}$ Fluo-3 and observed under the phase-contrast and fluorescence microscope. Scale bar, $20 \mu \mathrm{m}$. (d) YFP-ER/435S cells were pretreated with $10 \mu \mathrm{m}$ BAPTA plus $10 \mu \mathrm{m}$ BAPTA-AM and further treated with $5 \mathrm{~nm}$ bortezomib plus $30 \mu \mathrm{m}$ nutlin-3 for $16 \mathrm{~h}$. Treated cells were stained with $100 \mathrm{~nm}$ MTR and observed under phase-contrast and the fluorescence microscope. Scale bar, $20 \mu \mathrm{m}$.

(Figure 6b), suggesting that mitochondrial $\mathrm{Ca}^{2+}$ overload may contribute to bortezomib/nutlin-3-induced cell death. Interestingly, cellular detachment due to death, but not cellular vacuolation, was markedly inhibited by Ru360 pretreatment (Figure 6c). Fluorescence microscopy employing MTG and Rhod-2 showed that enhanced Rhod-2 fluorescence was co-localized with dilated mitochondria in MDA-MB 435S cells treated with bortezomib/nutlin-3 at $8 \mathrm{~h}$ post treatment (Figure 6d). Ru360 pretreatment effectively diminished this increase in mitochondrial $\mathrm{Ca}^{2+}$ levels, but did not affect the vacuolation induced by bortezomib/nultilin-3. In addition, Ru360 pretreatment did not affect the bortezomib/nultilin-3induced dilations of mitochondria or the ER, as shown by fluorescence microscopy of MTR-stained YFP-ER cells (Figure 6e). We also examined whether MCU knockdown reproduced the effect of RU360, and found that, similar to Ru360 pretreatment (Figure 6b), MCU knockdown slightly but significantly attenuated the cell death induced by bortezomib/ nutlin-3 (Figure 6f). Phase-contrast microscopy also showed that MCU knockdown reduced death-related cellular detachment but not vacuolation in bortezomib/nutlin-3-treated cells (Figure 6g). Furthermore, fluorescence microscopy using specific antibodies against PDI and COX II showed that the bortezomib/nutlin-3-induced dilations of the ER and mitochondria were not affected by MCU knockdown (Figure 6h). We also investigated whether the increase in mitochondrial $\mathrm{Ca}^{2+}$ depended on the increase in cytosolic $\mathrm{Ca}^{2+}$ levels observed during bortezomib/nutlin-3-induced paraptosis. Pretreatment with BAPTA plus BAPTA-AM markedly reduced the bortezomib/nutlin-3-indcued increase of Rhod-2 intensity (Figure 6i), suggesting that the bortezomib/nutlin-3-triggered increase in cytosolic $\mathrm{Ca}^{2+}$ levels may lead to MCU-mediated mitochondrial $\mathrm{Ca}^{2+}$ overload. Collectively, these results suggest that mitochondrial $\mathrm{Ca}^{2+}$ overload contributes to the cancer cell death induced by bortezomib/nutlin-3, but may not be a direct cause of vacuolation.

Next, we investigated the possible cause of the mitochondrial dilation seen during bortezomib/nutlin-3-indcued paraptosis. Previously, Soslau and Nass ${ }^{42}$ showed that ethidium bromide (EtBr) treatment induced the formation of MG, similar to bortezomib/nutlin-3 co-treatment. In addition, EtBr was shown to induce the mitochondrial unfolded protein response (mtUPR). ${ }^{43}$ Although the mechanisms underlying the mtUPR remain largely unknown, mitochondrial heat shock protein 70 (mtHSP70) has been proposed as a potential marker for this process. ${ }^{44,45}$ To investigate whether nutlin-3-induced mitochondrial dilation is associated with the mtUPR, we first tested whether EtBr treatment could induce mitochondrial dilation in MDA-MB 435S cells. Fluorescence microscopy of MTR-stained cells showed that treatment of MDA-MB 435S cells with $5 \mu \mathrm{g} \mathrm{ml}^{-1}$ EtBr-induced mitochondrial dilation (Figure 7a). Immunocytochemical analysis of mtHsp70 demonstrated that $\mathrm{EtBr}$ treatment increased the expression of mtHsp70 expression, and that this heat shock protein 
co-localized with COX II in dilated mitochondria (Figure 7b and $c$ ). These results suggest that the mtUPR may be induced during EtBr-induced mitochondrial dilation. Notably, nutlin-3 treatment also increased $\mathrm{mtHsp70}$ expression in dilated mitochondria (Figure $7 \mathrm{~b}$ and c). Next, we investigated whether $\mathrm{EtBr}$ could mimic the ability of nutlin-3 to sensitize resistant cancer cells to bortezomib-mediated cell death. We treated MDA-MB $435 \mathrm{~S}$ cells with $5 \mu \mathrm{g} \mathrm{ml}^{-1} \mathrm{EtBr}$ for 12 or $24 \mathrm{~h}$, refreshed the medium, and further treated the cells with $5 \mathrm{~nm}$ bortezomib for $24 \mathrm{~h}$. We found that this combined treatment with bortezomib and $\mathrm{EtBr}$ induced significantly more cell death than either treatment alone (Figure 7d). Observation of cellular
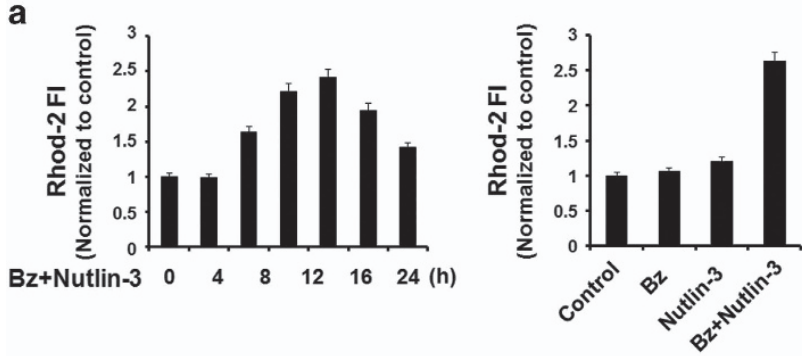

d

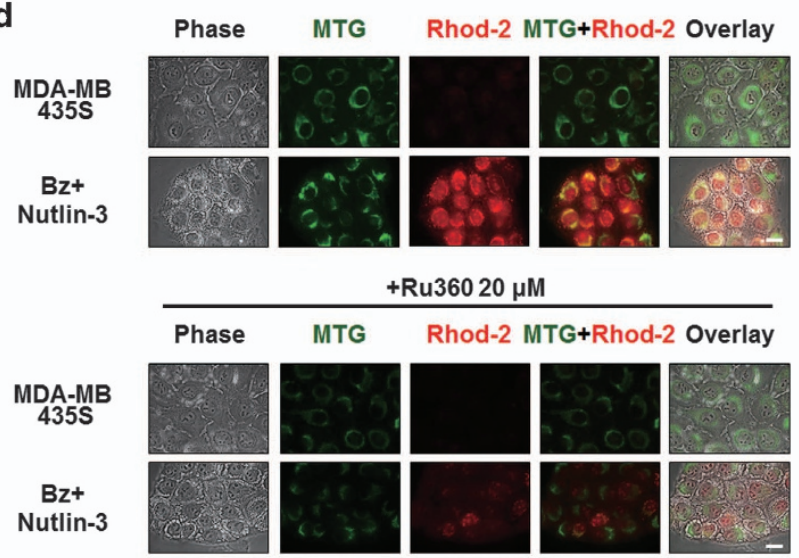

f

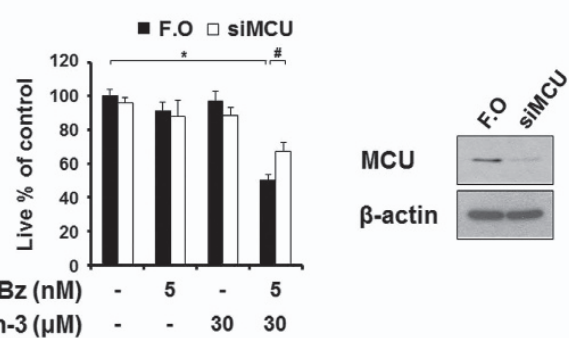

h

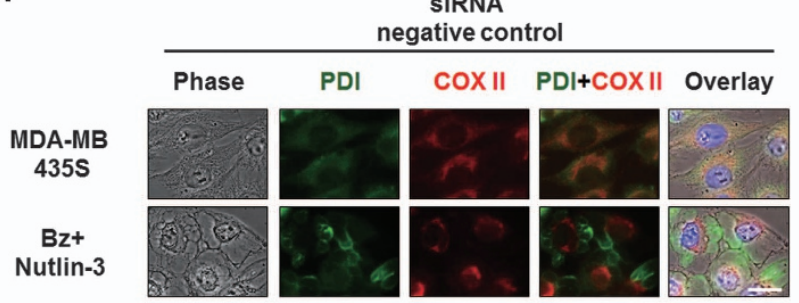

i

Bz+

Nutlin-3

BAPTA+

BAPTA-AM+

Bz+Nutlin-3 b
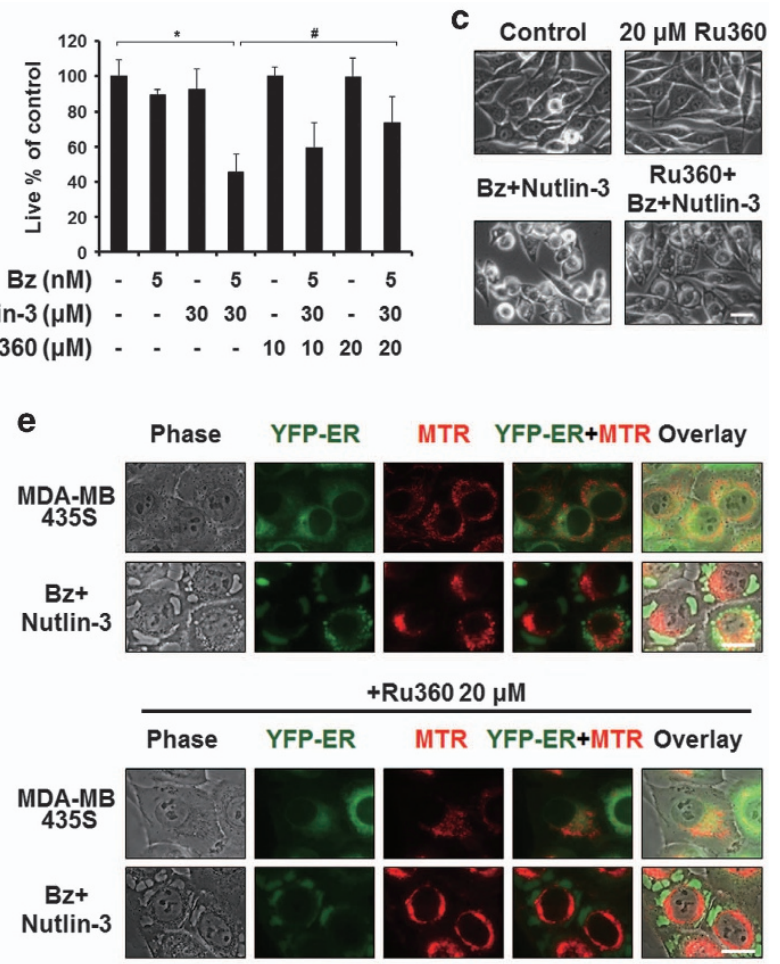

g
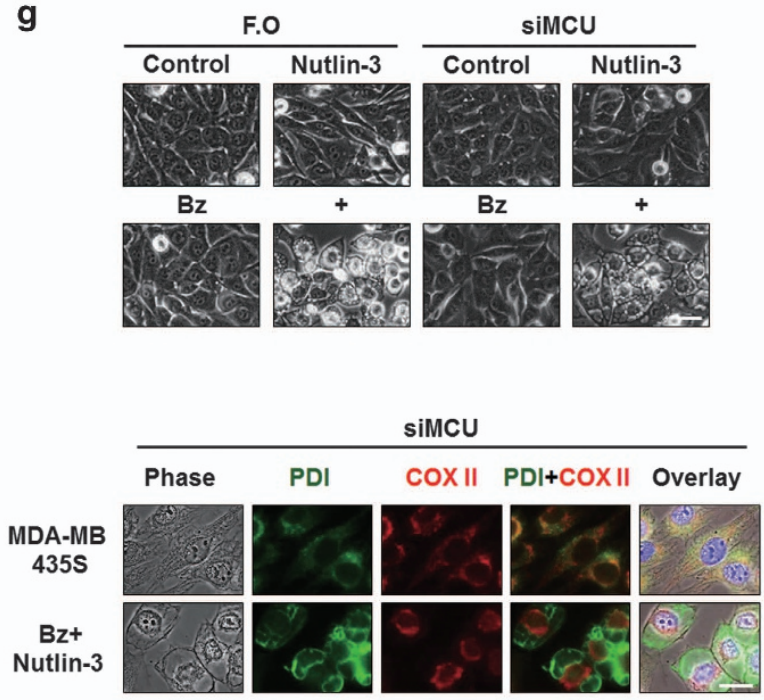
morphologies showed that bortezomib/EtBr, but not either agent alone, triggered notable cellular vacuolation (Figure 7e). Immunocytochemical analysis of PDI and COX II showed that bortezomib/EtBr also induced dilation of the ER and mitochondria prior to cell death (Figure 7f). Furthermore, not only bortezomib/EtBr but also bortezomib/nutlin-3 markedly increased the expression of $\mathrm{mtHsp} 70$ within the dilated mitochondria (Figure $7 \mathrm{~g}$ ). These results collectively suggest that nultin-3 may induce the accumulation of misfolded proteins within mitochondria, thereby contributing to mitochondrial dilation. The resulting mitochondrial stress may trigger the mtUPR in cancer cells. However, simultaneously and chronically imposed stress on the ER and mitochondria by bortezomib/nutlin-3 may result in irreversible failure in proteostasis, induction of terminal UPR and ultimately cell death, although its underlying mechanisms remain to be further explored.

\section{Bortezomib/nutlin-3 co-treatment induces proteostatic failure and subsequent ER and mitochondrial stress, potentially contributing to the dilations of these organelles} Finally, we investigated the relationship among the critical signals involved in the cell death induced by bortezomib/ nutlin-3. Since CHX pretreatment very effectively inhibited the dilations of both the ER and mitochondria (Figure 3d), we first examined the effect of CHX on the ER stress induced by bortezomib/nutlin-3. We found that CHX pretreatment very effectively blocked the co-treatment-induced accumulations of poly-ubiquitinated proteins and CHOP (Figure 8a). In addition, $\mathrm{CHX}$ pretreatment effectively attenuated the bortezomib/nutlin-3-induced increases in cytosolic and mitochondrial $\mathrm{Ca}^{2+}$ levels (Figure $8 \mathrm{~b}$ and $\mathrm{c}$ ) and abolished the bortezomib/nutlin-3-induced increase in mtHSP70 expression (Figure $8 \mathrm{~d}$ and e). Taken together, these results suggest that active protein synthesis is required for the bortezomib/nutlin3-induced dilations of the ER and mitochondria, and affects the critical signals for co-treatment-induced paraptosis. In addition, we found that shRNA-mediated CHOP knockdown markedly attenuated the bortezomib/nutlin-3 treatmentinduced increases in cytosolic and mitochondrial $\mathrm{Ca}^{2+}$ levels (Figure $8 \mathrm{f}$ and $\mathrm{g}$ ) but did not affect the accumulation of poly-ubiquitinated proteins (Figure $8 \mathrm{~h}$ ). This suggests that the accumulation of misfolded proteins in bortezomib/nutlin-3treated cells precedes the upregulation of CHOP. Interestingly, pretreatment with BAPTA plus BAPTA-AM markedly inhibited the bortezomib/nutlin-3-induced upregulation of CHOP but did not entirely inhibit the accumulation of poly-ubiquitinated proteins in co-treated cells (Figure 8i). These results suggest that bortezomib/nutlin-3 treatment induces proteasomal impairment and the subsequent accumulation of misfolded proteins. This may lead to increases in the levels of cytosolic $\mathrm{Ca}^{2+}$ and CHOP, which contribute to dilating the ER. In addition, a positive regulatory loop may exist between the increases in cytosolic $\mathrm{Ca}^{2+}$ and CHOP during bortezomib/nutlin-3-induced paraptosis.

In summary, we herein show that co-treatment with nutlin-3 overcomes bortezomib resistance in various p53-defective cancer cells by inducing paraptosis. During this process, ER dilation is critically promoted by proteasomal impairment and a subsequent positive regulatory loop between the induction of CHOP and the disruption of $\mathrm{Ca}^{2+}$ homeostasis, while mitochondrial dilation appears to be promoted by the stress caused by misfolded protein accumulation (Figure $8 \mathrm{j}$ ). Therefore, co-treatment with bortezomib and nultin-3 appears to lead to an irrecoverable failure in proteostasis that may be responsible for the severe physical and functional changes seen in the ER and mitochondria, leading to paraptotic cell death.

\section{DISCUSSION}

Although bortezomib has demonstrated promising anti-cancer effects in multiple myeloma and MCL, ${ }^{1,2}$ bortezomib-treated patients tend to acquire resistance to this agent, resulting in

Figure $6 \mathrm{MCU}$-mediated mitochondrial $\mathrm{Ca}^{2+}$ overload is important for the cell death but not for vacuolation induced by bortezomib/ nutlin-3. (a) MDA-MB 435S cells treated with $5 \mathrm{~nm}$ bortezomib and $30 \mu \mathrm{m}$ nutlin-3 for the indicated time points were stained with $1 \mu \mathrm{m}$ Rhod-2 and processed for FACS analysis. Rhod-2 fluorescence intensities (FI) in cells treated with 5 nm bortezomib plus $30 \mu \mathrm{m}$ nutlin-3 for the indicated time points were compared with those of untreated cells. (b) MDA-MB 435S cells were pretreated with or without Ru360 at the indicated concentrations and further treated with $5 \mathrm{~nm}$ bortezomib plus $30 \mu \mathrm{m}$ nutlin- 3 for $24 \mathrm{~h}$. Cellular viability was assessed using calcein-AM and EthD-1. Data represent the means \pm s.d. Kruskal-Wallis test was performed followed by Dunn's test. ${ }^{*} P<0.005$ vs untreated cells, ${ }^{\#} P<0.005$ vs cells treated with bortezomib/nultin-3. (c) MDA-MB 435S cells were untreated or treated with 5 nM bortezomib and $30 \mu \mathrm{m}$ nutlin-3 in the absence or presence of $20 \mu \mathrm{m}$ Ru360 for $24 \mathrm{~h}$. Cells were observed under the phase-contrast microscope. Scale bar, $10 \mu \mathrm{m}$. (d, e) MDA-MB 435S cells (d) or YFP-ER/435S cells (e) were treated with $5 \mathrm{~nm}$ bortezomib and $30 \mu \mathrm{m}$ nutlin-3 for $8 \mathrm{~h}$ in the absence or presence of Ru360. Treated cells were stained with $1 \mu \mathrm{m}$ Rhod-2 and $100 \mathrm{~nm}$ MTG (d) or stained with $100 \mathrm{~nm}$ MTR (e) and observed under the phase-contrast and fluorescence microscope. Scale bars, $20 \mu \mathrm{m}$. (f, g) MDA-MB 435S cells were transfected with the fluorescent oligonucleotide (FO) or a MCU-targeting siRNA (siMCU) for $24 \mathrm{~h}$ and further treated with $5 \mathrm{~nm}$ bortezomib plus $30 \mu \mathrm{m}$ nutlin-3 for $24 \mathrm{~h}$. (f) Cell viability was assessed using calcein-AM and EthD-1. Data represent the means \pm s.d. Kruskal-Wallis test was performed followed by Dunn's test. ${ }^{*} P<0.005$ vs cells transfected with FO, ${ }^{*} P<0.005$ vs cells transfected with FO and further treated with bortezomib/nutlin-3. Knockdown of MCU was confirmed by western blotting and $\beta$-actin was used as a loading control in western blots. (g) Transfected and treated cells were observed under the phase-contrast microscope. Scale bars, $10 \mu \mathrm{m}$. (h) Transfected cells were treated with $5 \mathrm{~nm}$ bortezomib plus $30 \mu \mathrm{m}$ nutlin-3 for $16 \mathrm{~h}$, fixed, and subjected for immunocytochemistry of COX II and PDI. Scale bars, $20 \mu \mathrm{m}$. (i) MDA-MB 435S cells were pretreated with or without $10 \mu \mathrm{m}$ BAPTA plus $10 \mu \mathrm{m}$ BAPTA-AM for 30 min and further treated with $5 \mathrm{~nm}$ bortezomib and $30 \mu \mathrm{m}$ nutlin-3 for $16 \mathrm{~h}$. Treated cells were stained with $1 \mu \mathrm{m}$ Rhod-2 and $100 \mathrm{~nm}$ MTG and observed under the phase-contrast and fluorescence microscope. Scale bar, $20 \mu \mathrm{m}$. 
a

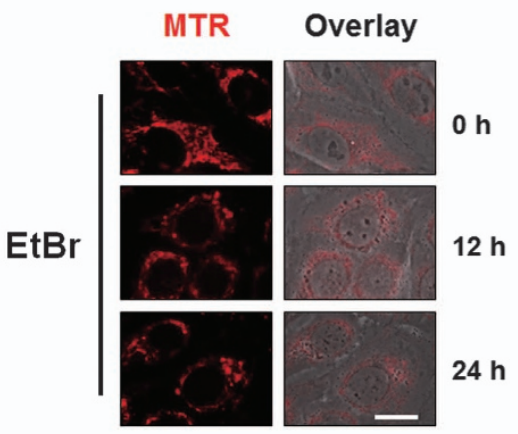

b

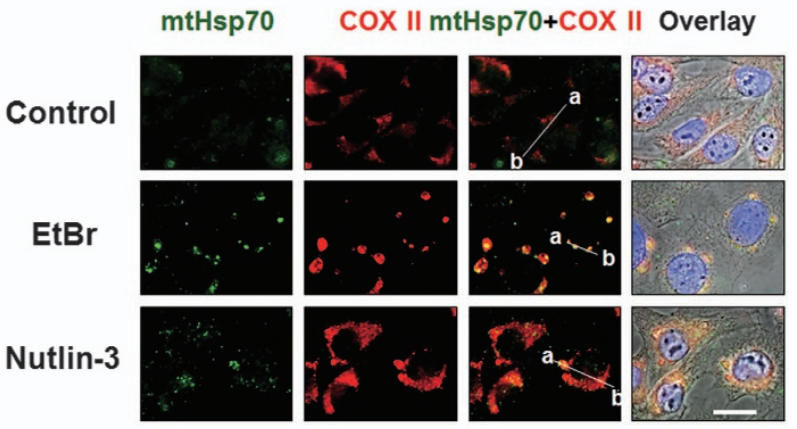

C

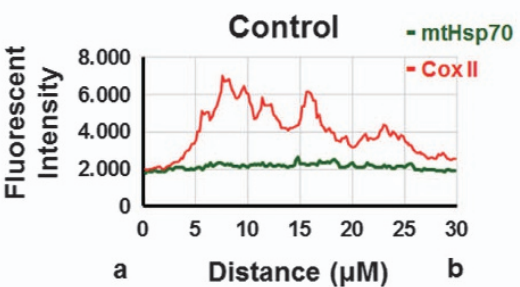

d

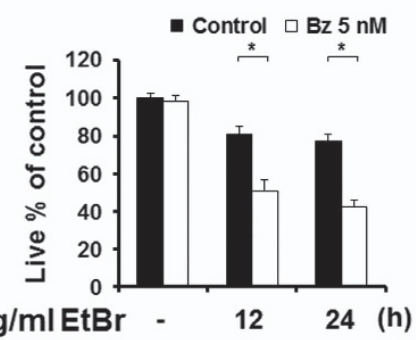

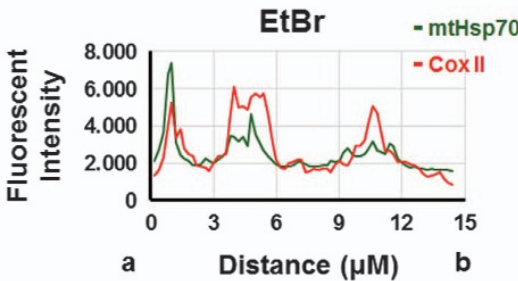

f

Control

$B z+E t B r$
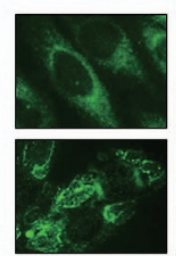
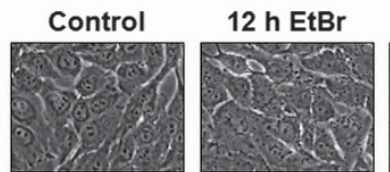

(w.o)

$+12 \mathrm{~h} \mathrm{Bz}$

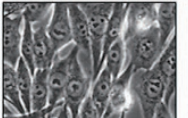

(w.o)
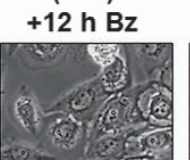

$24 \mathrm{~h} \mathrm{EtBr}$

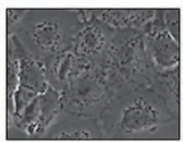

(w.o)

$+12 \mathrm{~h} \mathrm{Bz}$

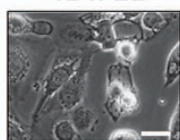

g

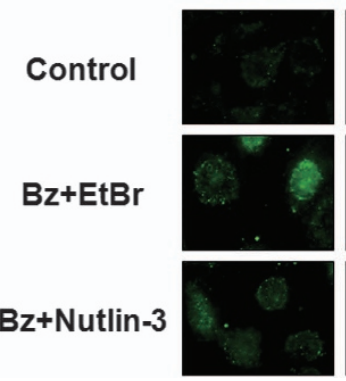

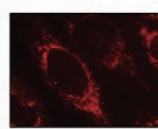
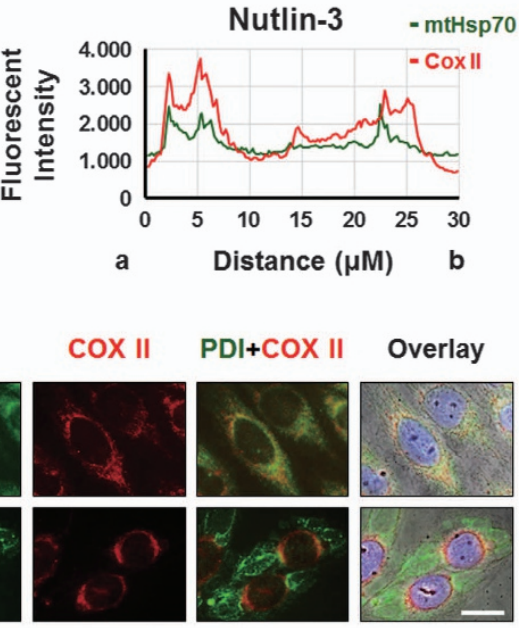

COX II PDI+COX II Overlay

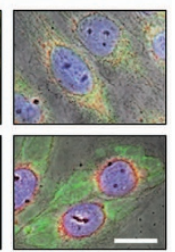

Figure 7 Bortezomib/nutlin-3 may trigger mitochondrial unfolded protein response (mtUPR) during mitochondrial dilation. (a) MDA-MB 435S cells treated with $5 \mu \mathrm{g} \mathrm{ml}^{-1} \mathrm{EtBr}$ for the indicated time points were stained with $100 \mathrm{~nm}$ MTR and observed under the phasecontrast and fluorescence microscope. Scale bars, $20 \mu \mathrm{m}$. (b) MDA-MB $435 \mathrm{~S}$ cells treated with $5 \mu \mathrm{g} \mathrm{ml}-1$ EtBr for $24 \mathrm{~h}$ or $30 \mu \mathrm{m}$ nutlin-3 for $16 \mathrm{~h}$ were fixed and subjected for immunocytochemistry of COX II and mtHsp70. Scale bars, $20 \mu \mathrm{m}$. (c) Plots of the intensity profile of a set of pixels distributed on a line drawn across the indicated mitochondria (shown in mtHsp70+COX II panels) are shown at different emission wavelengths corresponding to the signals of mtHsp70 (green) and COX II (red). (d-g) After treatment of MDA-MB 435S cells with $5 \mu \mathrm{g} \mathrm{ml}^{-1} \mathrm{EtBr}$ for the indicated time points, culture media were changed with the fresh ones and cells were further treated with $5 \mathrm{~nm}$ bortezomib for $24 \mathrm{~h}$. (d) Cell viability was assessed using calcein-AM and EthD-1. Data represent the means \pm s.d. One-way ANOVA and Bonferroni's post hoc test. ${ }^{*} P<0.005$ vs cells treated with EtBr alone for the indicated time points. (e) Treated cells were observed under the phase-contrast microscope. Scale bar, $10 \mu \mathrm{m}$. (f, $\mathbf{g})$ Treated cells were fixed, and subjected for immunocytochemistry of COX II and PDI (f) or COX II and mtHsp70 (g). In addition, immunocytochemistriy of COX II and mtHsp70 was performed in MDA-MB 435S cells treated with $5 \mathrm{~nm}$ bortezomib and/or $30 \mu \mathrm{m}$ nutlin-3 for $16 \mathrm{~h}$. Scale bars, $20 \mu \mathrm{m}$.

cancer recurrence and the failure of further cancer therapy. In addition, bortezomib has not shown a satisfactory therapeutic effect in solid tumors. ${ }^{4,5,46}$ Thus, researchers are currently seeking strategies to sensitize resistant cancer cells to this and other proteasome inhibitors.
Nutlin-3, which efficiently activates $\mathrm{p} 53,{ }^{6,7}$ was previously shown to increase the bortezomib sensitivity of multiple myeloma cells ${ }^{29,30} \mathrm{MCL}$ cells,,${ }^{10,31}$ and several epithelial cancer cell lines, including thyroid cancer cells, ${ }^{30}$ by enhancing apoptosis. In MCL cells with WT p53, the anti-cancer effects 
of bortezomib/nutlin-3 were proposed to involve the accumulation of $\mathrm{p} 53$ and the induction of p53-dependent proteins, including p21, MDM2, Puma and Noxa. ${ }^{10}$ In p53-mutant MCL cells, the cell death induced by bortezomib/nutlin-3 was postulated to involve the inductions of p73 and Noxa, plus the activations of Bax, Bak, caspase- 9 and caspase- $3 .{ }^{31}$ Critically, we herein show for the first time that bortezomib/nutlin-3 co-treatment synergistically kills various solid tumor cells that harbor non-functional p53 and are relatively resistant to bortezomib, and that this occurs via the induction of paraptosis.

a

Ub

CHOP

$\beta$-actin

$\mathrm{Bz}(\mathrm{nM})$

Nutlin-3 ( $\mu \mathrm{M})$
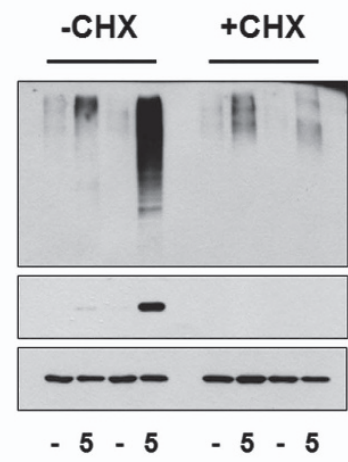

$-3030 \quad-3030$ b

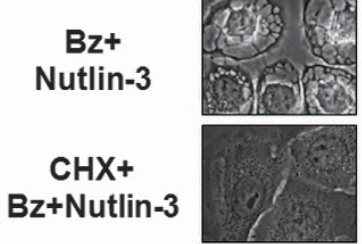

c

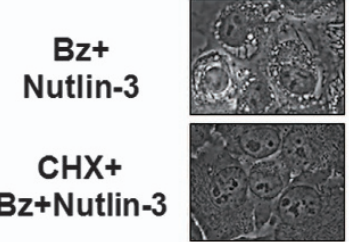

\section{Phase}

The cell death induced by bortezomib/nultin-3 in our system was accompanied by swelling and progressive fusion of the ER, along with mitochondrial dilation and eventual formation of MG. The mechanisms underlying paraptosis, particular the signals responsible for the dilation of the ER and/or mitochondria, are still not clearly understood. However, Mimnaugh et al. ${ }^{47}$ proposed that a proteasome-inhibition-triggered overload of misfolded proteins in the ER lumen could exert an osmotic pressure that draws water from the cytoplasm to distend the ER lumen. Consistent with this hypothesis, our previous studies showed that impairment of proteasome
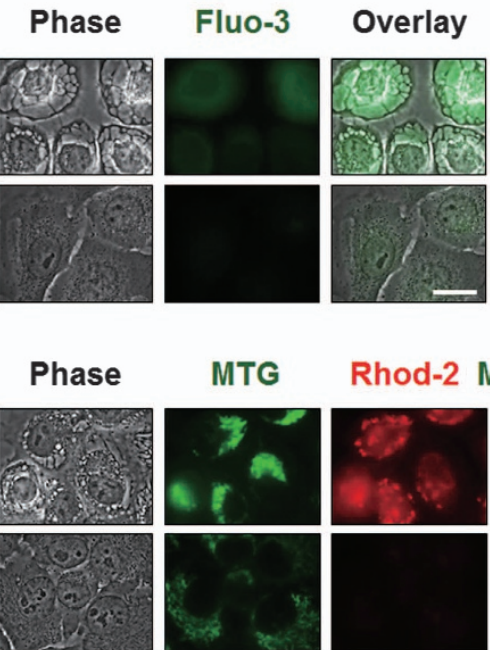

Rhod-2 MTG+Rhod-2 Overlay
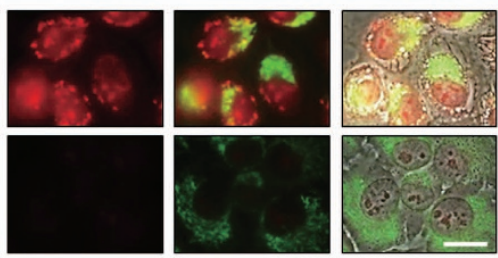

d

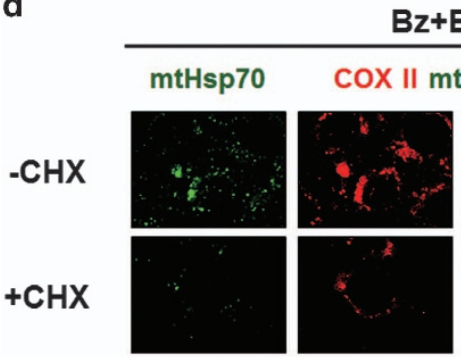

$\mathrm{Bz}+\mathrm{EtBr}$

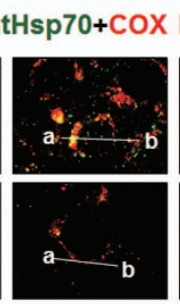

$B z+E t B r$

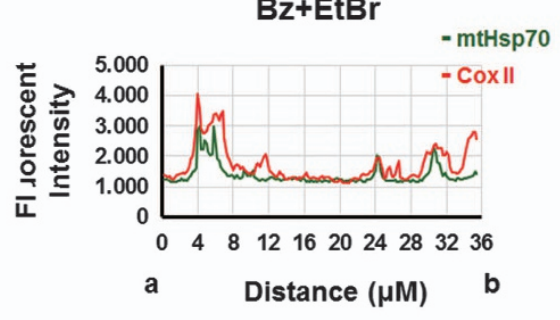

$\mathrm{CHX}+\mathrm{Bz}+\mathrm{EtBr}$

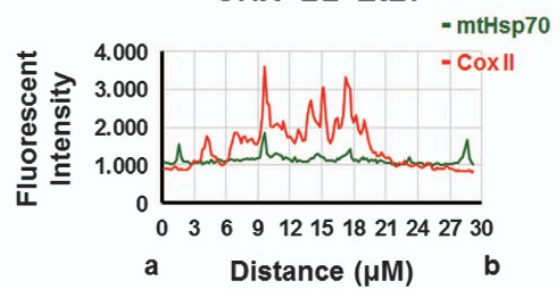

Bz+Nutlin-3

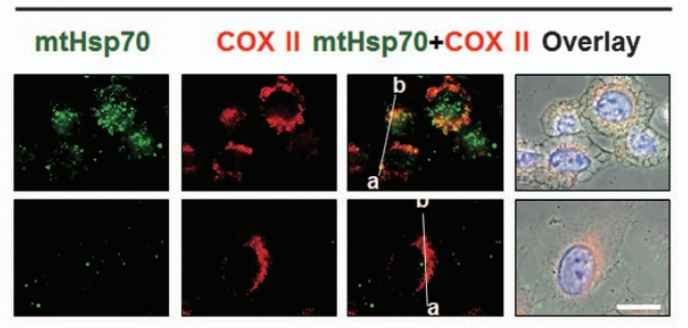

Bz+Nutlin-3

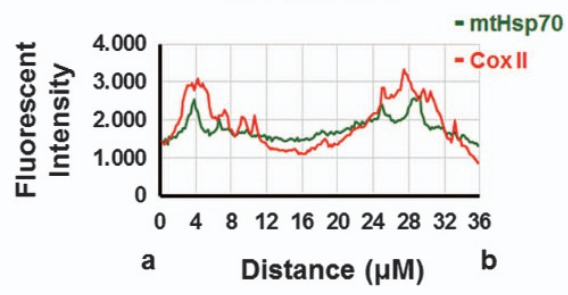

CHX+Bz+Nutlin-3

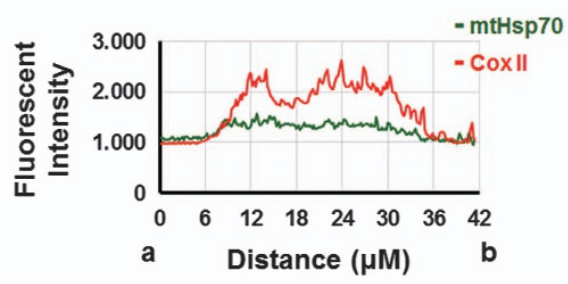


f
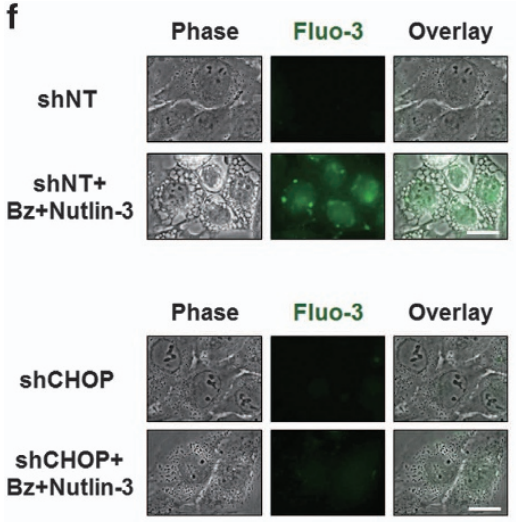

g

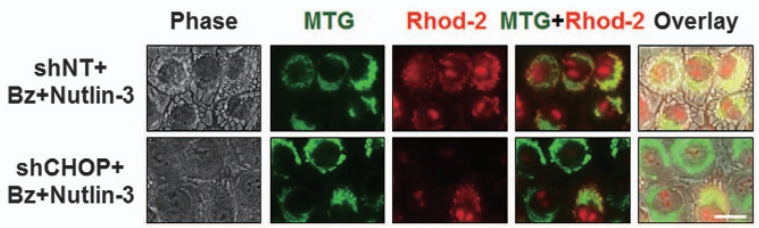

h

CHOP

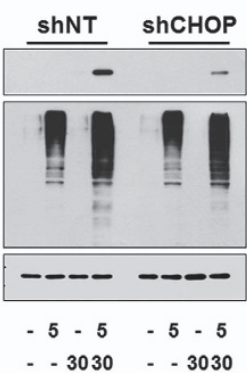

i

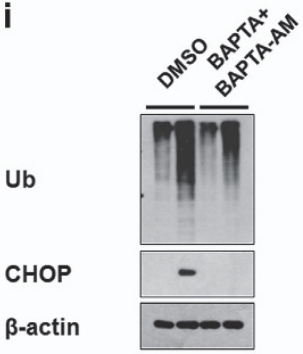

Bz+Nutlin-3 $\ldots++$

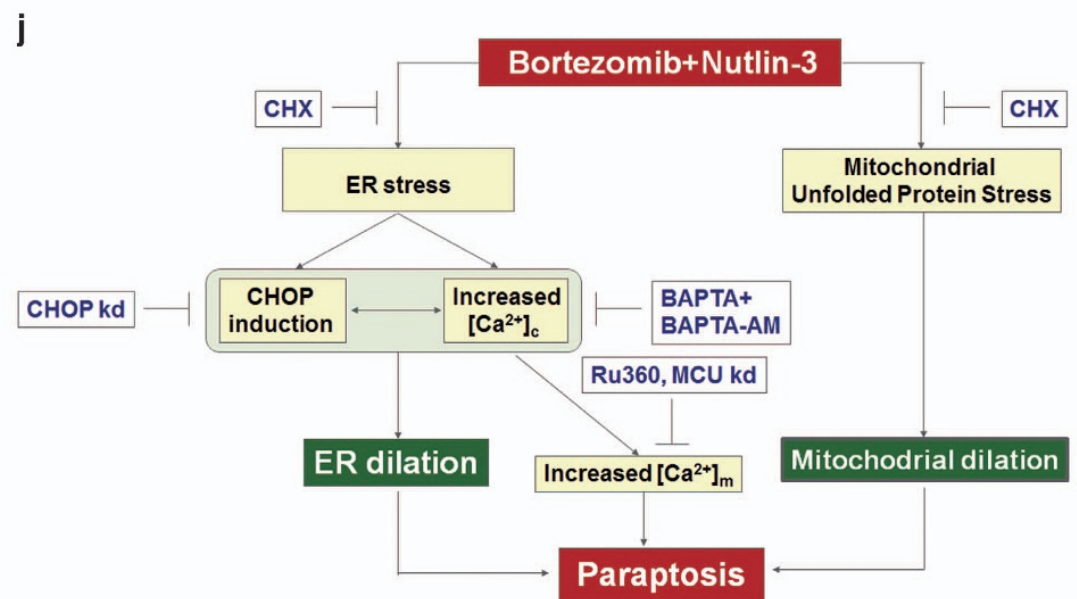

Figure 8 Upregulation of $\mathrm{CHOP}$ and the increase in $\mathrm{Ca}^{2+}$ levels precede the ER dilation induced by bortezomib/nutlin-3. (a) MDA-MB $435 \mathrm{~S}$ cells pretreated with or without $2 \mu \mathrm{m} \mathrm{CHX}$ and further treated with $5 \mathrm{~nm}$ bortezomib plus $30 \mu \mathrm{m}$ nutlin-3 for $16 \mathrm{~h}$. Cell extracts were prepared for the western blotting of ubiquitin and CHOP. $\beta$-actin was used as a loading control in western blots. (b, c) MDA-MB $435 \mathrm{~S}$ cells were pre-treated with or without $2 \mu \mathrm{m} \mathrm{CHX}$ for 30 min further treated with $5 \mathrm{~nm}$ bortezomib and $30 \mu \mathrm{m}$ nutlin-3 for $16 \mathrm{~h}$. (b) Treated cells were stained with $1 \mu \mathrm{m}$ Fluo-3 and observed under the phase-contrast and fluorescence microscope. Scale bar, $20 \mu \mathrm{m}$. (c) Treated cells were stained with $1 \mu \mathrm{M}$ Rhod-2 and $100 \mathrm{~nm}$ MTG and observed under the phase-contrast and fluorescence microscope. Scale bar, $20 \mu \mathrm{m}$. (d) MDA-MB 435S cells pretreated with or without $2 \mu \mathrm{m} \mathrm{CHX}$ followed by treatment with $5 \mu \mathrm{g} / \mathrm{ml} \mathrm{EtBr}$ for $24 \mathrm{~h}$. After culture media was changed with the fresh ones, cells were further treated with $5 \mathrm{~nm}$ bortezomib for $12 \mathrm{~h}$. Treated cells were fixed, and subjected for immunocytochemistry of COX II and mtHsp70. Scale bars, $20 \mu \mathrm{m}$. (e) Plots of the intensity profile of a set of pixels distributed on a lline drawn across the indicated mitochondria (as shown in mtHsp70+COX II panels) at differnet emission wavelengths corresponding to the signals of $\mathrm{mHHsp70}$ (green) and COX II (red). (f-h) MDA-MB 435S cells were infected with the lentivirus containing non-targeting (NT) shRNA or a CHOP-targeting shRNA (CHOP shRNA) for $24 \mathrm{~h}$. Infected cells were treated with $5 \mathrm{~nm}$ bortezomib and $30 \mu \mathrm{m}$ nutlin-3 for $16 \mathrm{~h}$. (f) Treated cells were stained with $1 \mu \mathrm{m}$ Fluo-3 and observed under the phase-contrast and fluorescence microscope. Scale bars, $20 \mu \mathrm{m}$. (g) Treated cells were stained with $1 \mu \mathrm{m}$ Rhod-2 and $100 \mathrm{~nm}$ MTG and observed under the phase-contrast and fluorescence microscope. Scale bars, $20 \mu \mathrm{m}$. (h) Cell extracts were prepared for western blotting of CHOP and ubiquitin. $\beta$-actin was used as a loading control in western blots. (i) MDA-MB 435S cells pretreated with $10 \mu \mathrm{M}$ BAPTA and $10 \mu \mathrm{M}$ BAPTA-AM were further treated with $5 \mathrm{~nm}$ bortezomib plus $30 \mu \mathrm{m}$ nutlin- 3 for $8 \mathrm{~h}$ and then western blotting of the indicated proteins was performed. $\beta$-actin was used as a loading control in western blots. (j) Hypothetical model of the underlying mechanism of paraptosis induced by bortezomib/nutlin3 in p53-defective cancer cells. Combination of bortezomib and nutlin-3 triggers ER stress induction, CHOP upregulation, and the increase in cytosolic $\mathrm{Ca}^{2+}$ levels, leading to ER dilation. In parallel to ER dilation, mitochondria are also dilated possibly via the mechanism related to mitochondrial unfolded protein stress, contributing to the paraptotic cell death induced by bortezomib/nutlin-3. Mitochondrial Ca ${ }^{2+}$ overload, which is preceded by increased cytosolic $\mathrm{Ca}^{2+}$, may also play a role in the cytotoxicity of bortezomib/nutlin-3.

activity is critical for the dilations of the ER seen during curcumin-, dimethoxycurcumin- and celastrol-induced paraptosis. ${ }^{18-21}$ In the present study, we show that nutlin-3 critically enhances bortezomib-mediated proteasome inhibition and ER stress, and that this critically contributes to dilating the ER. Co-treatment with nutlin-3 also markedly enhanced the bortezomib-mediated accumulation of poly-ubiquitinated proteins and CHOP. Moreover, CHOP knockdown effectively 
attenuated the ER dilation and cell death induced by bortezomib/nutlin-3, suggesting that the induction of CHOP is critical to this process. Han et al. ${ }^{48}$ recently reported that ATF4- and CHOP-mediated transcriptional regulation increases proteins synthesis, critically contributing to ER-stress-induced cell death. Therefore, it remains to be clarified whether the induction of $\mathrm{CHOP}$ observed in the present work contributes to ER dilation through the synthesis of specific protein(s) or a global increase in protein synthesis. Interestingly, we found that BAPTA-AM-mediated scavenging of intracellular $\mathrm{Ca}^{2+}$ also significantly inhibited the ER dilation and cell death induced by bortezomib/nutlin-3, and that the further addition of BAPTA enhanced this effect. These results indicate that disruption of $\mathrm{Ca}^{2+}$ homeostasis plays a key role in the ER dilation and cell death observed in the studied system. Since co-treated cells exhibited increased mitochondrial $\mathrm{Ca}^{2+}$ levels, in addition to increased cytosolic $\mathrm{Ca}^{2+}$ levels, we tested the functional significance of mitochondrial $\mathrm{Ca}^{2+}$ overload in the paraptosis induced by bortezomib/nutlin-3. Interestingly, inhibition of mitochondrial $\mathrm{Ca}^{2+}$ uptake by RU360 pretreatment or MCU knockdown significantly attenuated cell death by, but not the dilation of mitochondria and the ER, in bortezomib/nutlin3 -treated cells. This indicates that the induced mitochondrial $\mathrm{Ca}^{2+}$ overload may help shift the cellular balance toward cell death but may not be critically involved in mitochondrial dilation or MG formation in co-treated cells. Although the pathophysiological importance of MG still remains to be investigated, several possible mechanisms have been suggested to govern their formation, including swelling, the fusion of adjacent mitochondria and the suppression of mitochondrial division. ${ }^{49,50}$ The dilated mitochondria in co-treated cells were more than three times larger than normal mitochondria, ${ }^{50}$ suggesting that the mitochondrial dilation induced by bortezomib/nutlin-3 may not reflect simple mitochondrial swelling. When we investigated the possible involvement of various proteins associated with mitochondrial dynamics, we found that the protein levels of Fis1, MFN1 and MFN2 were not dramatically altered by treatment with bortezomib and/or nutlin-3 for $8 \mathrm{~h}$ (Supplementary Figure 3a). The protein levels of DRP1 were slightly increased by nutlin-3 treatment, but not by the combined treatment. In resolved lysates from untreated cells, OPA1 migrated as a complex mixture of at least five protein bands, possibly reflecting different isoforms and/or the presence of proteolytic processing. ${ }^{51}$ Nutlin-3 treatment resulted in the reduction of the long forms of OPA1, and the combined treatment further reduced them. Similar expression patterns of OPA1 were observed in T98G, DLD-1 and HeLa cells treated with nutlin-3 alone or with bortezomib/nutlin-3 (Supplementary Figure 3b). However, siRNA-mediated knockdown of OPA1 did not affect the cell death induced by bortezomib or bortezomib/nutlin-3 (Supplementary Figure 3c and d), suggesting that altered OPA1 expression may not be important for the cell death induced by the co-treatment. In addition, unlike knockdown of MCU, knockdown of Drp1, Fis1 or Mfn1 did not affect the cell death induced by bortezomib/nutlin-3. While the endoplasmic and cytosolic
UPRs have been extensively studied, the presence of a specific mtUPR has only recently emerged. ${ }^{52,53}$ The mtUPR is a form of retrograde signaling that contributes to the quality control of mitochondria by ensuring the functional integrity of the mitochondrial proteome. ${ }^{43}$ Mitochondria have dedicated molecular chaperones and proteases that promote proper protein folding, complex assembly and quality control. ${ }^{53}$ However, when misfolded proteins or unassembled complexes accumulate beyond the folding capacity, mitochondria and/or cells can experience altered proteostasis, damages and dysfunction. In the present study, we questioned whether the MG formation observed during bortezomib/nutlin-3-induced paraptosis was associated with the mtUPR. EtBr, which was previously shown to be an inducer of MG formation, ${ }^{42}$ is known to initiate the mtUPR. ${ }^{43,45,53}$ and increase the expression of mtHSP70, a candidate marker for the mtUPR. ${ }^{42,43,54}$ Since mitochondrial electron transport chain complexes rely on a precise stoichiometry between nuclear- and mtDNA-encoded proteins, ${ }^{55}$ inhibition of mitochondrial transcription and replication by $\mathrm{EtBr}$ causes folding defects or misassembly of mitochondrial protein complexes. ${ }^{54}$ Similar to the effect of EtBr, recombinant human HMGB1 (rhHMGB1) was recently reported to induce non-apoptotic cell death accompanied by formation of giant mitochondria in glioma cells. ${ }^{56}$ During this cell death, researchers observed depletion of mitochondrial DNA, severe damage of the mitochondrial proteome, and subsequent protein adduct formation within the mitochondria. These results further support the potential association between MG formation and the mtUPR. Here we found that not only EtBr but also nutlin-3 induced mitochondrial dilation and increased the expression of HSP70 in the dilated mitochondria of MDA-MB 435 S cells. Bortezomib/EtBr induced the dilation of both the ER and mitochondria in a manner similar to that seen in cells treated with bortezomib/nutlin-3. Furthermore, EtBr enhanced cancer cell death when combined with bortezomib, similar to the effect of nutlin-3. Taken together, these results suggest that nutlin-3-induced mitochondrial dilation may be closely associated with the accumulation of misfolded proteins within mitochondria and the subsequent induction of the mtUPR. Interestingly, nutlin-3 alone transiently induced mitochondrial dilation, while bortezomib alone triggered a very slight ER dilation (detectable only by electron microscopy). Therefore, we cannot exclude the possibility that the inductions of the mtUPR (by nutlin-3) and ER UPR (by bortezomib) may play adaptive and cytoprotective roles that explain the sub-lethal effects of either agent alone. In contrast, treatment with bortezomib and nultin-3 together may trigger terminal UPR by inducing simultaneous and sustained stresses in both mitochondria and the ER, leading to their severe and irreversible dilation and ultimate paraptotic cell death.

The present results obtained using isogenic lines of HCT116 cells differing in their p53 status showed that nutlin-3-mediated cytotoxicity requires WT p53. However, we further found that nutlin-3 effectively enhanced the death of HCT116 $\mathrm{p} 53^{-/-}$ cells (which were resistant to nutlin-3 alone) when combined with subtoxic doses of bortezomib. Nutlin-3 has a high affinity 
for the p53-binding pocket on the amino terminus of MDM2. ${ }^{6}$ RITA (reactivation of p53 and induction of tumor cell apoptosis), which is an another p53-reactivating small molecule, binds the amino terminus on p53 domain instead of on HDM2 protein. ${ }^{57}$ When we tested whether RITA mimicked the effect of nutlin-3 in our experimental system, we found that RITA alone dose-dependently enhanced cell death in HCT116 WT cells, but not in HCT116 $\mathrm{p} 53^{-/-}$cells, and that RITA co-treatment slightly increased bortezomib-induced cell death in HCT116 WT cells (Supplementary Figure 4a and b). In the latter effect, RITA resembled nutlin-3 (Figure $1 \mathrm{c}$ and $\mathrm{d}$ ). However, unlike nutlin-3 co-treatment, RITA co-treatment did not overcome the resistance of HCT116 $\mathrm{p} 53^{-/-}$cells to bortezomib (Supplementary Figure $4 \mathrm{a}$ and b). Also in contrast to the effect of nultin-3 co-treatment (Figure 1e-h), RITA did not noticeably increase the vacuolation and cell death of MDA-MB 435S cells when combined with various proteasome inhibitors, including bortezomib, carfilzomib and MG 132 (Supplementary Figure $4 \mathrm{c}$ and $\mathrm{d}$ ). These results suggest that the ability of nutlin-3 to sensitize cancer cells to proteasome inhibitors may be independent of its ability to block the p53-HDM2 interaction.

It is intriguing to consider how two distinct phenomena (apoptosis or paraptosis) can be triggered by the combination of bortezomib and nutlin-3. Although the mechanisms underlying these distinct effects remain to be further clarified, we speculate that the induction of different cell death modes following bortezomib/nutlin-3 treatment is likely to reflect both the cancer cell type (for example, hematopoietic or solid tumors) and the integrity of the apoptotic machinery (including the p53 status). While apoptosis is considered a major cell death mode underlying the cancer therapy of hematopoietic cancer cells, its contribution to treatment success in solid tumors has been debated. ${ }^{58,59}$ In addition, the ability of nutlin-3 to restore the apoptotic response is known to depend on p53. ${ }^{6}$ Therefore, while the absence of functional p53 may enable solid tumor cells to resist bortezomib/nutlin-3-induced apoptosis, these cancer cells may still be vulnerable to the induction of paraptosis by the same treatment. Our results suggested that the addition of nutlin-3 may allow the therapeutic dose of bortezomib to be reduced, which could potentially alleviate possible unwanted drug side effects in cancer cells with defective p53. In addition, co-treatment with nutlin-3 may extend the application of bortezomib to malignancies that show limited sensitivity to bortezomib-mediated apoptosis by inducing paraptosis, an alternative cell death. Improving our understanding of this alternative cell death mode may facilitate the design of novel therapeutics aimed at malignant cancer cells with defects in their apoptotic machineries.

\section{CONFLICT OF INTEREST}

The authors declare no conflict of interest.

\section{ACKNOWLEDGEMENTS}

This work was supported by the National Research Foundation of Korea (NRF) grants (Mid-Career Researcher Program No. 2015R1A2A2A1006966 and 2011-0030043 (SRC)) funded by the Korean government and a grant from the Korean Health Technology R\&D Project, Ministry of Health \& Welfare (HI14C2230).

1 Kane RC, Farrell AT, Sridhara R, Pazdur R. United States Food and Drug Administration approval summary: bortezomib for the treatment of progressive multiple myeloma after one prior therapy. Clin Cancer Res 2006; 12: 2955-2960.

2 Kane RC, Dagher R, Farrel A, Ko CW, Sridhara R, Justice R et al. Bortezomib for the treatment of mantle cell lymphoma. Clin Cancer Res 2007; 13: 5291-5294.

3 Ruschak AM, Slassi M, Kay LE, Schimmer AD. Novel proteasome inhibitors to overcome bortezomib resistance. J Natl Cancer Inst 2011; 103: 1007-1017.

4 Dispenzieri A, Jacobus S, Vesole DH, Callandar N, Fonseca R, Greipp PR. Primary therapy with single agent bortezomib as induction, maintenance and re-induction in patients with high-risk myeloma: results of the ECOG E2A02 trial. Leukemia 2010; 24: 1406-1411.

5 Kale AJ, Moore BS. Molecular mechanisms of acquired proteasome inhibitor resistance. J Med Chem 2012; 55: 10317-10327.

6 Vassilev LT, Vu BT, Graves B, Carvajal D, Podlaski F, Filipovic Z et al. In vivo activation of the p53 pathway by small-molecule antagonists of MDM2. Science 2004; 303: 844-848.

7 Vassilev LT. MDM2 inhibitors for cancer therapy. Trends Mol Med 2007; 13: 23-31.

8 Shen H, Maki CG. Pharmacologic activation of p53 by small-molecule MDM2 antagonists. Curr Pharm Des 2011; 17: 560-568.

9 Lau LM, Nugent JK, Zhao X, Irwin MS. HDM2 antagonist nutlin-3 disrupts p73-HDM2 binding and enhances p73 function. Oncogene 2008; 27: 997-1003.

10 Tabe Y, Sebasigari D, Jin L, Rudelius M, Davies-Hill T, Miyake $\mathrm{K}$ et al. MDM2 antagonist nutlin-3 displays antiproliferative and proapoptotic activity in mantle cell lymphoma. Clin Cancer Res 2009; 15: 933-942.

11 Supiot S, Hill RP, Bristow RG. Nutlin-3 radiosensitizes hypoxic prostate cancer cells independent of p53. Mol Cancer Ther 2008; 7: 993-999.

12 Peirce SK, Findley HW. The MDM2 antagonist nutlin-3 sensitizes p53-null neuroblastoma cells to doxorubicin via E2F1 and TAp73. Int J Oncol 2009; 34: 1395-1402.

13 Zheng T, Yin D, Lu Z, Wang J, Li Y, Chen X et al. Nutlin-3 overcomes arsenic trioxide resistance and tumor metastasis mediated by mutant p53 in hepatocellular carcinoma. Mol Cancer 2014; 13: 133.

14 Schmitt CA. Senescence, apoptosis and therapy-cutting the lifelines of cancer. Nat Rev Cancer 2003; 3: 286-295.

15 Wattel E, Preudhomme C, Hecquet B, Vanrumbeke M, Quesnel B, Dervite I et al. p53 mutations are associated with resistance to chemotherapy and short survival in hematologic malignancies. Blood 1994; 84: 3148-3157.

16 Sperandio S, de Belle I, Bredesen DE. An alternative, nonapoptotic form of programmed cell death. Proc Natl Acad Sci USA 2000; 97: 14376-14381.

17 Sperandio S, Poksay K, de Belle I, Lafuente MJ, Liu B, Nasir J et al. Paraptosis: mediation by MAP kinases and inhibition by AIP-1/Alix. Cell Death Differ 2004; 11: 1066-1075.

18 Yoon MJ, Kim EH, Lim JH, Kwon TK, Choi KS. Superoxide anion and proteasomal dysfunction contribute to curcumin-induced paraptosis of malignant breast cancer cells. Free Radic Biol Med 2010; 48: 713-726.

19 Yoon MJ, Kim EH, Kwon TK, Park SA, Choi KS. Simultaneous mitochondrial $\mathrm{Ca}(2+)$ overload and proteasomal inhibition are responsible for the induction of paraptosis in malignant breast cancer cells. Cancer Lett 2012; 324: 197-209.

20 Yoon MJ, Lee AR, Jeong SA, Kim YS, Kim JY, Kwon YJ et al. Release of $\mathrm{Ca}^{2+}$ from the endoplasmic reticulum and its subsequent influx into mitochondria trigger celastrol-induced paraptosis in cancer cells. Oncotarget 2014; 5: 6816-6831.

21 Yoon MJ, Kang YJ, Lee JA, Kim IY, Kim MA, Lee YS et al. Stronger proteasomal inhibition and higher $\mathrm{CHOP}$ induction are responsible for more effective induction of paraptosis by dimethoxycurcumin than curcumin. Cell Death Dis 2014; 5: e1112. 
22 Kar R, Singha PK, Venkatachalam MA, Saikumar P. A novel role for MAP1 LC3 in nonautophagic cytoplasmic vacuolation death of cancer cells. Oncogene 2009; 28: 2556-2568.

23 Singha PK, Pandeswara S, Venkatachalam MA, Saikumar P. Manumycin A inhibits triple-negative breast cancer growth through LC3-mediated cytoplasmic vacuolation death. Cell Death Dis 2013; 4: e457.

24 Lee D, Kim IY, Saha S, Choi KS. Paraptosis in the anti-cancer arsenal of natural products. Pharmacol Ther 2016; 162: 120-133.

25 Lee WJ, Chien MH, Chow JM, Chang JL, Wen YC, Lin YW et al. Nonautophagic cytoplasmic vacuolation death induction in human PC-3M prostate cancer by curcumin through reactive oxygen species -mediated endoplasmic reticulum stress. Sci Rep 2015; 5: 10420.

26 Li B, Zhao J, Wang CZ, Searle J, He TC, Yuan CS et al. Ginsenoside Rh2 induces apoptosis and paraptosis-like cell death in colorectal cancer cells through activation of p53. Cancer Lett 2011; 301: 185-192.

27 Bury M, Girault A, Megalizzi V, Spiegl-Kreinecker S, Mathieu V, Berger W et al. Ophiobolin A induces paraptosis-like cell death in human glioblastoma cells by decreasing BKCa channel activity. Cell Death Dis 2013; 4: e561.

28 Hoa N, Myers MP, Douglass TG, Zhang JG, Delgado C, Driggers L et al. Molecular mechanisms of paraptosis induction: implications for a non-genetically modified tumor vaccine. PLoS ONE 2009; 4: e4631.

29 Saha MN, Jiang H, Jayakar J, Reece D, Branch DR, Chang H. MDM2 antagonist nutlin plus proteasome inhibitor velcade combination displays a synergistic anti-myeloma activity. Cancer Biol Ther 2010; 9: 936-944.

30 Ooi MG, Hayden PJ, Kotoula V, McMillin DW, Charalambous E, Daskalaki E et al. Interactions of the $\mathrm{Hdm} 2 / \mathrm{p} 53$ and proteasome pathways may enhance the antitumor activity of bortezomib. Clin Cancer Res 2009; 15: 7153-7160.

31 Jin L, Tabe $\mathrm{Y}$, Kojima K, Zhou Y, Pittaluga S, Konopleva $\mathrm{M}$ et al. MDM2 antagonist Nutlin-3 enhances bortezomib-mediated mitochondrial apoptosis in TP53-mutated mantle cell lymphoma. Cancer Lett 2010; 299: 161-170.

32 Gartel AL, Feliciano C, Tyner AL. A new method for determining the status of p53 in tumor cell lines of different origin. Oncol Res 2003; 13: 405-408.

33 Villalonga-Planells R, Coll-Mulet L, Martinez-Soler F, Castano E, Acebes JJ, Gimenez-Bonafe $\mathrm{P}$ et al. Activation of p53 by nutlin-3a induces apoptosis and cellular senescence in human glioblastoma multiforme. PLOS ONE 2011; 6: e18588.

34 Li Y, Mao Y, Brandt-Rauf PW, Williams AC, Fine RL. Selective induction of apoptosis in mutant p53 premalignant and malignant cancer cells by PRIMA-1 through the c-Jun-NH2-kinase pathway. Mol Cancer Ther 2005; 4: 901-909.

35 Hoppe-Seyler F, Butz K. Repression of endogenous p53 transactivation function in HeLa cervical carcinoma cells by human papillomavirus type 16 E6, human mdm-2, and mutant p53. J Virol 1993; 67: 3111-3117.

36 Rampias T, Sasaki C, Weinberger P, Psyrri A. E6 and E7 gene silencing and transformed phenotype of human papillomavirus 16-positive oropharyngeal cancer cells. J Natl Cancer Inst 2009; 101: 412-423.

37 Komarov PG, Komarova EA, Kondratov RV, Christov-Tselkov K, Coon JS, Chernov MV et al. A chemical inhibitor of p53 that protects mice from the side effects of cancer therapy. Science 1999; 285: 1733-1737.

38 Sperandio S, Poksay KS, Schilling B, Crippen D, Gibson BW, Bredesen DE. Identification of new modulators and protein alterations in non-apoptotic programmed cell death. J Cell Biochem 2010; 111: 1401-1412.

39 Nishitoh $\mathrm{H}$. CHOP is a multifunctional transcription factor in the ER stress response. J Biochem 2012; 151: 217-219.

40 De Stefani D, Rizzuto R. Molecular control of mitochondrial calcium uptake. Biochem Biophys Res Commun 2014; 449: 373-376.

41 De Stefani D, Patron M, Rizzuto R. Structure and function of the mitochondrial calcium uniporter complex. Biochim Biophys Acta 2015; 1853: 2006-2011.

42 Soslau G, Nass MM. Effects of ethidium bromide on the cytochrome content and ultrastructure of $\mathrm{L}$ cell mitochondria. J Cell Biol 1971; 51: 514-524.
43 Yoneda T, Benedetti C, Urano F, Clark SG, Harding HP, Ron D. Compartment-specific perturbation of protein handling activates genes encoding mitochondrial chaperones. J Cell Sci 2004; 117: 4055-4066.

44 Haynes CM, Petrova K, Benedetti C, Yang Y, Ron D. ClpP mediates activation of a mitochondrial unfolded protein response in $C$. elegans. Dev Cell 2007; 13: 467-480.

45 Al-Furoukh N, lanni A, Nolte H, Holper S, Kruger M, Wanrooij S et al. ClpX stimulates the mitochondrial unfolded protein response (UPRmt) in mammalian cells. Biochim Biophys Acta 2015; 1853: 2580-2591.

46 Chen D, Frezza M, Schmitt S, Kanwar J, Dou QP. Bortezomib as the first proteasome inhibitor anticancer drug: current status and future perspectives. Curr Cancer Drug Targets 2011; 11: 239-253.

47 Mimnaugh EG, Xu W, Vos M, Yuan X, Neckers L. Endoplasmic reticulum vacuolization and valosin-containing protein relocalization result from simultaneous hsp90 inhibition by geldanamycin and proteasome inhibition by velcade. Mol Cancer Res 2006; 4: 667-681.

48 Han J, Back SH, Hur J, Lin YH, Gildersleeve R, Shan J et al. ER-stress-induced transcriptional regulation increases protein synthesis leading to cell death. Nat Cell Biol 2013; 15: 481-490.

49 Wakabayashi T. Megamitochondria formation-physiology and pathology. J Cell Mol Med 2002; 6: 497-538.

50 Teranishi M, Karbowski M, Kurono C, Soji T, Wakabayashi T. Two types of the enlargement of mitochondria related to apoptosis: simple swelling and the formation of megamitochondria. J Electron Microsc 1999; 48: 637-651.

51 Ishihara N, Fujita Y, Oka T, Mihara K. Regulation of mitochondrial morphology through proteolytic cleavage of OPA1. EMBO J 2006; 25: 2966-2977.

52 Zhao Q, Wang J, Levichkin IV, Stasinopoulos S, Ryan MT, Hoogenraad NJ. A mitochondrial specific stress response in mammalian cells. EMBO $\mathrm{J}$ 2002: 21: 4411-4419.

53 Haynes CM, Ron D. The mitochondrial UPR-protecting organelle protein homeostasis. J Cell Sci 2010; 123: 3849-3855.

54 Hayashi J, Tanaka M, Sato W, Ozawa T, Yonekawa H, Kagawa $\mathrm{Y}$ et al. Effects of ethidium bromide treatment of mouse cells on expression and assembly of nuclear-coded subunits of complexes involved in the oxidative phosphorylation. Biochem Biophys Res Commun 1990; 167: 216-221.

55 Taanman JW. The mitochondrial genome: structure, transcription, translation and replication. Biochim Biophys Acta 1999; 1410: 103-123.

56 Gdynia G, Keith M, Kopitz J, Bergmann M, Fassl A, Weber AN et al. Danger signaling protein HMGB1 induces a distinct form of cell death accompanied by formation of giant mitochondria. Cancer Res 2010; 70: 8558-8568.

57 Saha MN, Jiang H, Mukai A, Chang H. RITA inhibits multiple myeloma cell growth through induction of p53-mediated caspase-dependent apoptosis and synergistically enhances nutlin-induced cytotoxic responses. Mol Cancer Ther 2010; 9: 3041-3051.

58 de Bruin EC, Medema JP. Apoptosis and non-apoptotic deaths in cancer development and treatment response. Cancer Treat Rev 2008; 34: 737-749.

59 Mansilla S, Llovera L, Portugal J. Chemotherapeutic targeting of cell death pathways. Anticancer Agents Med Chem 2012; 12: 226-238.

\section{This work is licensed under a Creative Commons}

Attribution-NonCommercial-ShareAlike

4.0

International License. The images or other third party material in this article are included in the article's Creative Commons license, unless indicated otherwise in the credit line; if the material is not included under the Creative Commons license, users will need to obtain permission from the license holder to reproduce the material. To view a copy of this license, visit http:// creativecommons.org/licenses/by-nc-sa/4.0/

Supplementary Information accompanies the paper on Experimental \& Molecular Medicine website (http://www.nature.com/emm) 